\title{
A Conditional Moment Closure Study of Chemical Reaction Source Terms in SCCI Combustion
}

\author{
James Jalal Behzadi ${ }^{1}$ (D) Mohsen Talei ${ }^{2}$. \\ Michele Bolla ${ }^{3}$ - Evatt R. Hawkes ${ }^{1,4}$. Tommaso Lucchini ${ }^{5}$. \\ Gianluca D'Errico ${ }^{5} \cdot$ Sanghoon Kook $^{1}$
}

Received: 28 June 2016 / Accepted: 10 June 2017 / Published online: 3 July 2017

(C) Springer Science+Business Media B.V. 2017

\begin{abstract}
The objective of this study is to evaluate conditional moment closure (CMC) approaches to model chemical reaction rates in compositionally stratified, autoigniting mixtures, in thermochemical conditions relevant to stratified charge compression ignition (SCCI) engines. First-order closure, second-order closure and double conditioning are evaluated and contrasted as options in comparison to a series of direct numerical simulations (DNSs). The two-dimensional (2D) DNS cases simulate ignitions in SCCI-like thermochemical conditions with compositionally stratified $n$-heptane/air mixtures in a constant volume. The cases feature two different levels of stratification with three mean temperatures in the negative-temperature coefficient (NTC) regime of ignition delay times. The first-order closure approach for reaction rates is first assessed using hybrid DNS-CMC a posteriori tests when implemented in an open source computational fluid dynamics (CFD) package known as OpenFOAM ${ }^{\circledR}$. The hybrid DNS-CMC a posteriori tests are not a full CMC but a DNS-CMC hybrid in that they compute the scalar and velocity fields at the DNS resolution, thus isolating the first-order reaction rate closure model as the main source of modelling error (as opposed to turbulence model, scalar probability density function model, and scalar dissipation rate model). The hybrid DNS-CMC a posteriori test reveals an excellent
\end{abstract}

Evatt R. Hawkes

evatt.hawkes@unsw.edu.au

James Jalal Behzadi

J.Behzadi@unsw.edu.au

1 School of Mechanical and Manufacturing Engineering, The University of New South Wales, Sydney, NSW 2052, Australia

2 Department of Mechanical Engineering, University of Melbourne, VIC 3010, Australia

3 Department of Mechanical and Process Engineering, ETH Zürich, 8092 Zürich, Switzerland

4 School of Photovoltaic and Renewable Energy Engineering, The University of New South Wales, Sydney, NSW 2052, Australia

5 Dipartimento di Energia, Politecnico di Milano, 20156 Milan, Italy 
agreement between the model and DNS for the cases with low levels of stratification, whereas deviations from the DNS are observed in cases which exhibit high level of stratifications. The a priori analysis reveals that the reason for disagreement is failure of the first-order closure hypothesis in the model due to the high level of conditional fluctuations. Second-order and double conditioning approaches are then evaluated in a priori tests to determine the most promising path forwards in addressing higher levels of stratification. The a priori tests use the DNS data to compute the model terms, thus directly evaluating the model assumptions. It is shown that in the cases with a high level of stratification, even the second-order estimation of the reaction rate source term cannot provide a reasonably accurate closure. Double conditioning using mixture-fraction and sensible enthalpy, however, provides an accurate first-order closure to the reaction rate source term.

Keywords CMC $\cdot$ OpenFOAM $\cdot$ HCCI $\cdot$ SCCI $\cdot$ DNS $\cdot$ Composition stratification

\section{Introduction}

Modern internal combustion (IC) engines need to meet increasingly stringent regulations on globally significant pollutants like $\mathrm{CO}_{2}$ and urban pollutants such as soot, $\mathrm{NO}_{x}$, particulate matter, unburned hydrocarbons, and $\mathrm{CO}$. In response to these issues, low temperature combustion (LTC) engines are emerging as an alternative to traditional spark-ignited (SI) and diesel engines. Homogeneous charge compression ignition (HCCI) is a subclass of LTC engines which may reduce both urban and global emissions. An HCCI engine operates using a lean premixed fuel/air charge at an elevated compression ratio (CR) compared to SI engines. Due to the highly diluted nature of mixture, combustion takes place at a lower temperature compared with a conventional diesel engine, resulting in low emissions of NOx and soot. Thanks to its higher CR, lack of throttling losses, and low combustion temperature, HCCI engines are also more efficient than conventional SI engines [1-3].

Despite their advantages over SI and diesel engines, commercialisation of HCCI combustion has so far been impeded by some technical challenges, with two of them being more pressing: (1) lack of ignition timing control over wide engine speed-load ranges; and (2) excessive pressure rise rate (PRR) at high load conditions. The first challenge is due to the fact that combustion in an HCCI engine is mostly driven by chemical kinetics, and is therefore controlled by the chemical properties of fuel as well as the temperature and pressure history of the fuel-air mixture. This makes it difficult to exercise direct control over combustion timing in HCCI engines compared with SI and diesel engines. The second challenge is attributed to the homogeneous state of fuel/air mixture prior to autoignition, which results in nearly simultaneous ignition of the charge and very high heat release rate (HRR) at high load conditions. This translates into high PRR which ultimately could damage the engine when combined with undesired ignition timing [1-4].

Stratification may address the PRR issues where inhomogeneities in the mixture temperature or composition, or both, are used to create a sequential combustion that spreads the heat release over a longer autoignition event. While thermal stratification of the charge may effectively control ignition timing and spread out the HRR leading to a smooth combustion process, in practice, exploiting thermal stratification for HRR control can be challenging $[1,5,6]$. As an alternative, fuel stratification has been used, leading to concepts known by several names, but here referred to as stratified charge compression ignition (SCCI) combustion. This technique provides a better control over ignition timing compared with thermal 
stratification. One way to achieve stratification in fuel/air composition is to employ a twostage injection strategy $[2,4,7]$. In the first stage a lean homogeneous background charge is prepared, usually using port fuel injection (PFI) or very early direction injection (DI). In the second stage, the remaining fuel (up to 60 percent of the total fuel volume [4]) is provided by one or more direct injection(s) during the late compression stroke and/or near TDC in the early expansion stroke. The level of stratification can be increased by retarding the injection and/or by increasing the portion of DI fuel. Due to its potential to solve problems with ignition timing and high PRR, SCCI combustion is still an active research area [1-19].

To provide a physical insight into the ignition behaviour under HCCI and SCCI combustion, direct numerical simulation (DNS) has been used, e.g. [20-36]. Most of these works, however, study thermal stratification of HCCI combustion, e.g. [20-27, 36], and it is only in recent years that a few studies attend to fuel stratification [28-35]. In the DNS works, instead of solving governing equations for a realistically sized engine, an affordable computational domain, usually in two dimensions, is generally initialised such that the integral time scales in this domain approximately match those of the TDC conditions in a real $\mathrm{HCCI} / \mathrm{SCCI}$ engine. Periodic boundary conditions are employed to simulate the constant volume autoignition in the bulk of the TDC in a real engine. DNS studies of fuel stratification involve various fuels such as hydrogen [28], $n$-heptane [29-31], dimethyl-ether (DME) [32, 33, 35], and biodiesel [34]. One common finding in most of these DNS works is identification of two dominant modes of combustion: deflagration and spontaneous ignition. The deflagration mode prevails when the role of molecular transport (conduction and diffusion) becomes significant compared to the chemical reaction; whereas the spontaneous mode corresponds to simultaneous ignition events that are only coupled through pressure with negligible molecular transport. The DNS data demonstrated that, on the one hand, the deflagration mode was predominant when the stratification levels are high in which the HRR is spread out. On the other hand, the spontaneous ignition prevails in cases with a low level of stratification which featured a high HRR, e.g. [29, 30]. Although DNS provides detailed understanding of autoignition under HCCI/SCCI conditions, it can not be used for design purposes of realistic engine sizes due to its prohibitive computational costs. Therefore, a number of combustion models have been developed that are computationally more affordable.

The multi-zone model is a common approach toward modelling combustion under HCCI conditions, e.g. [37]. In this approach some homogeneous reactors (called zones) are considered to represent the in-cylinder conditions. These zones are only coupled through pressure and the mixing between them is not included. Therefore, the model is only valid in the spontaneous ignition mode, and a poor performance is reported when the level of stratifications increases and the role of mixing becomes significant [38].

HCCI combustion has also been modelled using flamelet-based approaches. Pitsch and co-workers [39] developed an enthalpy-based transient flamelet model and validated their model against DNS data for thermally stratified HCCI. Later on, the same group extended the model to include compositional stratification as well [38], and validated their results against 2D DNS of SCCI-like conditions where fuel stratification and temperature stratification were uncorrelated. Recently, Im and co-workers [40] have coupled a spray-flamelet model with a computational fluid dynamics (CFD) code to study the injection timing under SCCI conditions in 2D. The spray-flamelet model makes use of the mixture-fraction as the conditioning variable. When compared with available experimental data the results from the flamelet model is superior to those of multi-zone for higher levels of stratification, which is attributed to the ability of flamelet approach to capture the small scale transport processes. 
More expensive models such as the probability density function (PDF) approach have also been used for modelling HCCI combustion [41, 42]. This method involves solution of the PDF transport equation in the composition or velocity-composition space using Eulerian fields or Lagrangian particles. Zhang et al. [41] implemented the PDF model in a 3D CFD code to study the turbulence/chemistry interaction (TCI) in an HCCI engine. Although their practice was general (due to the spatial terms in the PDF equations) and could highlight the effect of TCI by comparing the results of the PDF solver with the finite-volume solver, the computational cost for detailed chemistry and complicated 3D geometries would be very high. Bisetti et al. [42] considered a homogeneous transported PDF (ignoring the spatial terms) to simulate HCCI combustion and compared their results against DNS. Their results showed a good agreement with temporal trace of HRR and moments of temperature and hydroxyl radical mass fraction.

As an alternative to the PDF method, a much more computationally affordable yet mathematically rigorous technique, referred to as the conditional moment closure (CMC), is also a candidate. The CMC model was originally developed for non-premixed combustion by Klimenko [43] and Bilger [44]. In this method a set of transport equations for the conditional moments of the species mass fractions are solved [45]. In the past 10 years, the CMC approach has been successfully utilised to model various non-premixed flames, for instance bluff body flames [46-49], jet flames [50-59], hood fires [60, 61], and has also been extended to premixed flames [62-68]. CMC fundamentally works by conditioning the state composition vector on a variable or variables upon which it principally depends, which allows the impact of state-vector fluctuations to be significantly reduced in the closure of reaction rates. For sufficiently small conditional fluctuations, experience has shown that first-order $\mathrm{CMC}$, where the conditional mean reaction rate is evaluated directly as a function of the conditional mean thermochemical state vector, is sufficient [46-48, 50-56, 60-76]. For higher levels of fluctuations, either second-order CMC, e.g. [49, 57-59, 77], or double conditioning, e.g. [78, 79], have been proposed.

Most relevant to the present work are CMC studies involving autoignition. In the last decade a significant body of work has appeared applying CMC to diesel-engine relevant problems, which progressed from studies of spray autoignition [69-71], to application in real engines [72-75], and has also demonstrated successful predictions of soot formation $[70,76]$. Also there are some fundamental studies of non-premixed diesel-like autoignition using DNS with simplified [80-82] or detailed chemistry [83]. However, although it also involves fuel stratification, SCCI combustion is unlike diesel engine combustion in that there is not expected to be a mixing-controlled burn but rather the combustion occurs principally in a premixed mode.

There have been very few works devoted to CMC for essentially premixed autoignitions, such as are expected in SCCI. In ignition-driven problems, conditioning on a variable that maps well to ignition delay is desirable. Following this strategy, in recent previous works performed by our group [67, 68], a CMC model was developed to model thermally stratified mixtures under HCCI conditions using total enthalpy as a conditioning variable. It was shown that for low levels of stratification the model had an excellent performance. As the level of stratification increased the performance deteriorated. It was also shown the presence of large conditional fluctuations as a result of the predominant deflagration combustion mode was the reason for the weak performance of the CMC model at the highly stratified conditions. A detailed analysis of the second moment equations revealed that the underlying reason for generation of the conditional fluctuations is strong correlation between dissipation rate fluctuations and conditional mass fraction fluctuations. 
The present paper differs from our previous studies $[67,68]$ in that we now consider CMC modelling of cases featuring compositional stratification. Compositional stratification is an essential feature of SCCI, given that it is this stratification that is intentionally used to introduce a range of mixtures, which ignite at different times and thus reduce the PRR and/or provide ignition-timing control. However, to the best of our knowledge, no DNS studies of CMC for compositionally stratified ignitions relevant to $\mathrm{HCCI} / \mathrm{SCCI}$ combustion yet exist [80-83].

As earlier mentioned, in our previous modelling of thermally stratified ignition $[67,68]$, we argued that total enthalpy was a good choice for the conditioning variable because it mapped well to ignition delay and thus the state vector of different mixtures. However, in the present model, while composition stratification naturally results in a connected thermal stratification, due to the heat of evaporation of the fuel and due to differing temperature rise via compression of different mixtures, mixture-fraction is the natural choice of a conditioning variable.

With the above context, the first objective of this article is to evaluate the first-order CMC approach to model reaction rates in compositionally stratified autoignitions relevant to SCCI conditions. This will be achieved first by hybrid DNS-CMC a posteriori comparisons ${ }^{1}$ to a series of two-dimensional (2D) DNS cases modelling constant volume, compositionally stratified autoignitions of $n$-heptane. The DNS cases are set up in an attempt to mimic engine SCCI conditions in terms of the temperature, pressure, mean equivalence ratio, turbulence timescale, and stratification levels. The two-dimensional assumption implies that the DNS cases are not completely representative of the in-cylinder processes in an engine, but 3D simulations in the considered conditions were not feasible. Therefore, similar to several other works considering HCCI/SCCI-relevant conditions, we adopt the two-dimensional assumption in the DNS [20-34]. Another limitation of the present study is the lack of considering large-scale flow geometry effects, such as any residual large-scale statistically inhomogeneous structure left over from fuel injection, swirl, tumble, and squish flows, and the influence of walls. Since these effects occur on scales corresponding to the large-scale geometry, they are beyond possible consideration with DNS at the present time, due to the significant separation between such large scales and the small dissipative ones. Still, it is the authors' opinion that fundamentally oriented DNS studies, such as the present one, can provide useful input to an overall model development and validation approach, which must ultimately end with testing and use in an engine.

The a posteriori modelling is based on a fully coupled hybrid DNS-CMC solver, in which the conditional statistics are spatially zero-dimensional (like the DNS). To isolate only the issues around the conditioning closure of reaction rates, following previous work in the group on thermally stratified ignitions $[67,68]$, the CFD side of the model is deliberately run at the DNS resolution, which obviates the need for scalar dissipation rate (SDR), PDF models and turbulence models, thus removing issues in these sub-models as a possible source of modelling error. It is fully noted and acknowledged that the present work

\footnotetext{
${ }^{1}$ For clarity the hybrid DNS-CMC a posteriori and a priori concepts are defined here:
}

- a posteriori: is referred to when the CFD-CMC model is integrated in time according to the model equations starting from the DNS initial condition. The results are then compared to DNS data.

- a priori: is referred to when the DNS data are post-processed on their own without actually running the CMC model. 
does not consider a complete CMC model, which would require in addition to the reaction model we test, models for the above-mentioned quantities. Similar to the situation for thermally stratified ignition $[67,68]$, we will demonstrate in this article that the first-order closure of reaction rates performs well for low stratification levels, but that higher levels of stratification eventually cause an increase of conditional fluctuations and thus a breakdown of the first-order closure assumption. In this context, two approaches to solve this problem, namely second-order CMC and double conditioning, are then investigated and compared $a$ priori to provide guidance for future $\mathrm{CMC}$ development at higher stratification levels.

The paper is organised as follows. Section 2 presents the numerical methodologies for the CMC solver, and introduces the DNS and their parameters. Section 3 presents results focussing first on the hybrid DNS-CMC a posteriori predictions with the first-order model, and second on a priori analysis of the DNS to determine promising directions for future development. The paper concludes in Section 4.

\section{Numerical methodology}

In this section, the first-order CMC model equation is formally presented. Then the DNS solver utilised to generate the data for a priori analyses is introduced. The section is closed with the details of the test cases and initial conditions.

\subsection{Conditional moment closure (CMC)}

The spatially zero-dimensional CMC approach is developed for a statistically homogeneous system, i.e. one in which the statistics of the scalars involved have no spatial dependence. Put another way, the statistical ensemble considered is the whole domain of the DNS; in an engine application it would be the whole internal region of the cylinder. Local fluctuations can still occur but the assumption is under OD-CMC that these can be collapsed completely by variations of the conditioning variable. The transport equation for the mass fraction of a given species under the assumption of unity Lewis number can be written as $\rho D Y / D t-$ $\nabla \cdot\left(\rho D_{t h} \nabla Y\right)=\dot{\omega}$, where $\rho$ is density, $t$ is time, $D / D t$ is the material derivative, $D_{t h}$ is the thermal diffusivity, and $\dot{\omega}$ is the reaction rate source term. Furthermore, the transport equation of the mixture-fraction, $Z$, is expressed as

$$
\rho \frac{D Z}{D t}-\nabla \cdot\left(\rho D_{t h} \nabla Z\right)=0 .
$$

The conditional mean of a scalar, $\phi$ is denoted here as $\langle\phi \mid \eta\rangle=\phi_{\eta}$ where $\eta$ is the sample variable of $Z$ space. The conditional Favre mean of $\phi$ is defined as $\langle\rho \phi \mid \eta\rangle / \rho_{\eta}=\tilde{\phi}_{\eta}$. Accordingly the scalar $\phi$ may be decomposed into a conditional Favre mean and a conditional fluctuation component, viz. $\phi=\tilde{\phi}_{\eta}+\phi^{\prime \prime}$. To facilitate the computation, since in the present study the boundary conditions of mixture-fraction are not zero and one and also change with time, a normalised mixture-fraction is adopted

$$
Z_{\text {norm }}(x, t)=\frac{Z(x, t)-Z_{\min }(t)}{Z_{\max }(t)-Z_{\min }(t)}
$$

where $Z_{\min }$ and $Z_{\max }$ are only a function of time. Their difference is denoted here as

$$
\Delta \Theta(t) \equiv Z_{\max }(t)-Z_{\min }(t),
$$


If $\xi$ represents the sample variable in $Z_{\text {norm }}$ space, using the decomposition method, the normalised form of the spatially zero-dimensional CMC equation with unity Lewis number assumption can be derived, viz.

$$
\frac{\partial \tilde{Y}_{\xi}}{\partial t}=-\tilde{v}_{\xi} \frac{\partial \tilde{Y}_{\xi}}{\partial \xi}+\frac{1}{(\Delta \Theta)^{2}} \tilde{N}_{\xi} \frac{\partial^{2} \tilde{Y}_{\xi}}{\partial \xi^{2}}+\frac{\dot{\omega}_{\xi}}{\rho_{\xi}},
$$

where

$$
\tilde{v}_{\xi}=-\frac{1}{\Delta \Theta}\left[\frac{\partial Z_{\min }}{\partial t}+\frac{\left\langle\rho Z_{\text {norm }} \mid \xi\right\rangle}{\rho_{\xi}} \frac{\partial(\Delta \Theta)}{\partial t}\right],
$$

Note that normalisation is not necessary to our approach; it is simply a matter of convenience in avoiding to apply a boundary condition on a time-varying location in mixture-fraction space and to reduce the computational cost so that only the mixture-fraction ranges that actually exist are gridded at any one time. In a practical implementation, a non-normalised version would probably be preferred, to avoid the need for models for $Z_{\min }$ and $Z_{\max }$. In Eq. 4 the terms involving $Y^{\prime \prime}$, where $Y^{\prime \prime}=Y-\tilde{Y}_{\xi}$ is the Favre conditional fluctuation, are neglected following the primary closure hypothesis [45]. Also $N \equiv D_{t h}(\nabla Z)^{2}$ is defined as scalar dissipation rate and $\tilde{N}_{\xi}$ is its conditional Favre mean with respect to the normalised mixture-fraction. The convective term (in mixture-fraction space) on the right hand side of Eq. 4 does not normally appear in the conventional formulations of the CMC equation based on the mixture-fraction; this is due to conditioning on $Z_{\text {norm }}$ rather than $Z$.

It is important to note here a consequence of the choice of the Reynolds-averaged, spatially zero-dimensional framework adopted. The averaging operation when applied to the DNS removes all spatial structure of statistics. If, however, an LES framework were adopted, and a filter size smaller than the DNS domain were used, the filtered statistics would retain a spatial dependence and a CMC spatial mesh to deal with the corresponding transport of conditional moments would be required. This would be likely to improve results, with greater improvement expected for smaller LES filter sizes; however the computational cost would be orders of magnitude more than the methodology proposed here.

\subsection{Hybrid DNS-CMC in OpenFOAM framework (OF-CMC model)}

OpenFOAM ${ }^{\circledR}$ is an open source CFD package written in $\mathrm{C}++$ language. It offers transient implicit compressible solvers within framework of the PISO algorithm for pressuremomentum coupling $[84,85]$. It reads in the mesh in structured or unstructured format and discretises governing transport equations using finite volume approach [86]. OpenFOAM ${ }^{\circledR}$ uses Open-MPI libraries as its parallelisation infrastructure. The CMC model in Eq. 4 is implemented into OpenFOAM ${ }^{\circledR}$. The implementation is referred to as OF-CMC here, for brevity.

In the present model, OF-CMC solves the transport equations for momentum, total enthalpy (chemical + sensible), and the mixture-fraction at DNS grid resolution. As discussed earlier, this fine CFD grid resolution is deliberately employed in order to focus exclusively on the modelling issues surrounding the conditioning closure. This means in the present work turbulence is not modelled and only molecular diffusion is considered (no turbulent diffusion modelling). As a result, some additional submodels that would normally be required are not needed. For instance if the DNS resolution is not chosen, a model is needed for $\tilde{N}_{\xi}$ to solve Eq. 4, or as another example the PDF of mixture-fraction needs to be modelled. The choice of DNS grid resolution minimises the adverse effects that the aforementioned sub-models may have on the results of the combustion model. 
In Fig. 1, a flowchart of the implementation is illustrated. After all flow variables are solved in the CFD solver, including the mixture-fraction in Eq. 1, $\tilde{Y}_{\xi}$ for each species is updated in the CMC solver using Eq. 4. In Equation 4, the scalar dissipation rate is conditionally averaged over the considered statistical ensemble, i.e. the entire domain at one time instant. This is consistent with the mixture-fraction PDF defined over the same ensemble. The same applies to all other conditional average quantities needed in the CMC side (Fig. 1). Then using $\psi=\delta(Z(x, t)-\xi)$, the fine-grained PDF, $Y$ of that species on the CFD grid is interpolated depending on the local value of $Z$, viz.

$$
Y(x, t)=\delta(Z(x, t)-\xi) \tilde{Y}_{\xi}
$$

Use of the Dirac delta-PDF is justifiable as a deliberate feature of our hybrid DNS-CMC model because, as noted above, we deliberately resolve the conditioning variable enough so that we do not need a model for the PDF, in order to focus on other parts of the closure assumptions. ${ }^{2}$ This completes one time-step iteration of OF-CMC. ${ }^{3}$ The computation is parallelised, both in the CFD domain and in the CMC solver. A two-step operator splitting technique is utilised for efficient solution of Eq. 4 in the CMC solver:

1. In the first step, the reaction rate term is integrated in parallel. For this purpose the conditional bins are uniformly distributed among available processors. Upon completion, the solution is synced among all the processors. Depending on the case a speed-up factor of 20-30 was gained. OpenFOAM's native ODE-solver known as SIBS (semiimplicit Burlish-Stoer) is used to integrate the reaction rates. Since SIBS is an implicit solver, it requires provision of Jacobians. The Jacobians are numerically evaluated by a perturbation method using a first-order accurate forward difference formula. Also SIBS requires a derivatives routine where it evaluates the reaction rates. The conditional reaction rate, $\overline{\dot{\omega}}_{\xi}$, is evaluated using a first-order closure hypothesis, i.e. $\overline{\dot{\omega}}_{\xi} \simeq \dot{\omega}\left(\tilde{Y}_{\xi}, \tilde{T}_{\xi}, \bar{p}\right)$. The chemical mechanism used in this study was available as a Fortran subroutine which was compiled and linked with OpenFOAM.

2. In the second step the non-stiff terms, as shown in Fig. 1, are integrated in serial. In this step all the processors obtain their own solution of the CMC equation. Alternatively, one core could solve the equation and then the results could be synced with other cores. The non-stiff terms are integrated implicitly using OpenFOAM's linear LU solver. The convective term in Eq. 4 is discretised using first-order upwind scheme. Higher order schemes such as total variation diminishing (TVD) were also examined which led to the same results. The diffusion term is discretised using a second-order central difference approach. A uniform CMC mesh with $200+2$ bins are used for discretisation. ${ }^{4}$ Two ghost nodes are employed on each end of the mesh to avoid special treatment of the boundary nodes. The value of ghost nodes are approximated by a Neumann boundary condition from the adjacent internal nodes which are in turn updated in step 1

\footnotetext{
${ }^{2}$ Note that instead of feeding back the mean mass fractions, the density and other required properties such as the specific heats, viscosity, etc could be determined in the CMC domain and fed back. This would make the calculation cheaper but as a matter of convenience it is not implemented this way.

${ }^{3}$ The CMC solver may be invoked before the CFD solver, alternatively. For small enough time steps both approaches converge to the same solution.

${ }^{4}$ The number of bins was determined by a series of sensitivity tests not shown here.
} 


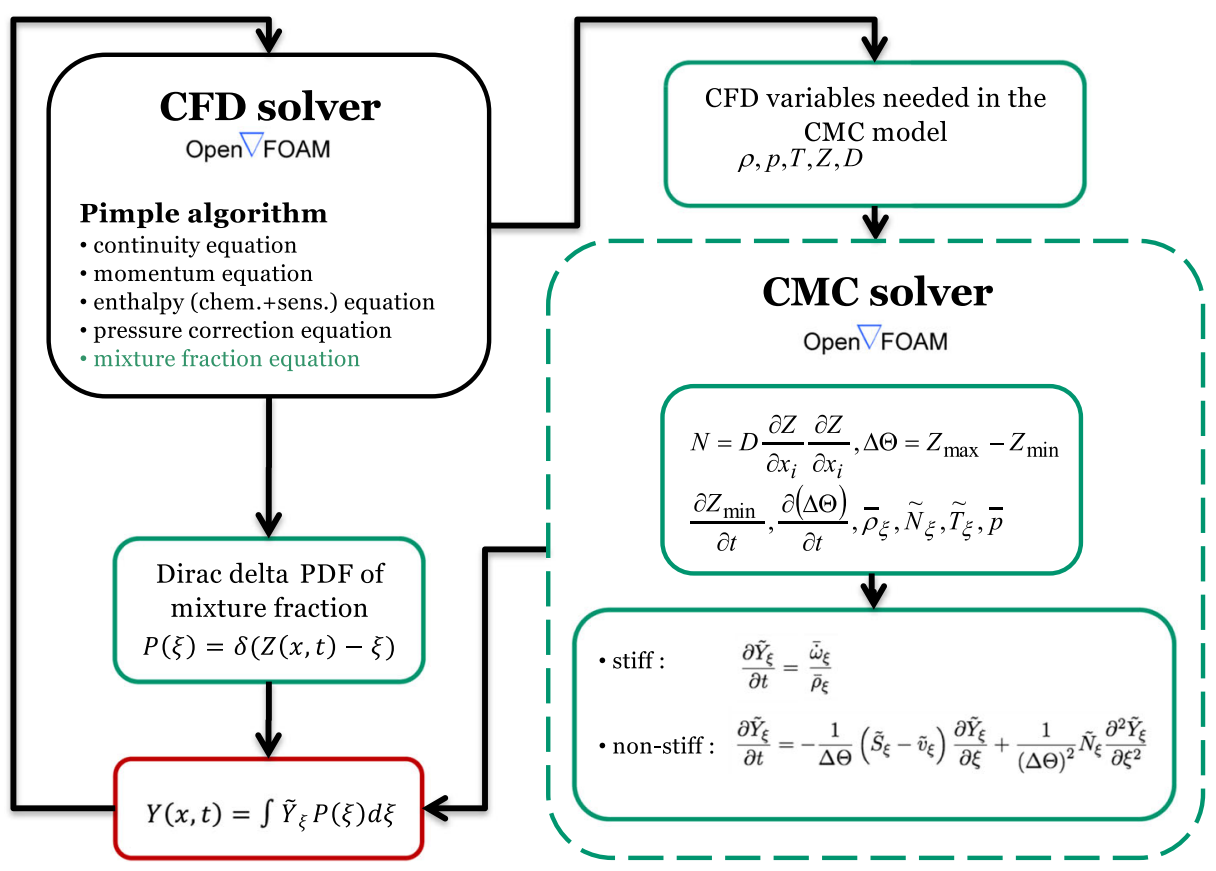

Fig. 1 The OF-CMC algorithm

above [87]. Having fixed the value of the ghost nodes ${ }^{5}$, the internal nodes are integrated implicitly to find the solution of the CMC equation for this time-step iteration.

The viscosity is found using Sutherland's formula. The diffusion coefficient in the total enthalpy transport equation is found by assuming $\operatorname{Pr}=0.705$. The same diffusion coefficient is used for transport of the mixture-fraction to be consistent with Le $=1$ assumption made in derivation of the CMC equation.

\subsection{The DNS solver}

The DNSs model a series of compositionally stratified ignitions of $n$-heptane in air. The intention of the DNSs are to represent, canonically and within computational constraints, the near top-dead centre (TDC) ignition of a small region within the bulk gas in the cylinder of an SCCI engine. They are very similar to previously reported DNS in Ref. [29], so only a

\footnotetext{
${ }^{5}$ In effect, the ghost nodes hold the value of boundary nodes from reaction step. While in pinciple, the value of ghost nodes can be transported to the internal nodes via diffusion and convection, in the present cases here they will not affect the solution in the internal nodes. On the boundary nodes at $\xi=0$ and $\xi=1$ the value of $N$ is fixed at zero by definition, therefore, no diffusion will take place from ghost nodes to internal nodes. As for convection, the $\tilde{v}_{\xi}$ in Eq. 5 is an outgoing velocity at both boundaries. At the left boundary, $\tilde{v}_{\xi}$ is negative (an outgoing wave from right to left), because at $\xi=0$ the velocity becomes $\tilde{v}_{\xi}=-\left(\partial Z_{\min } / \partial t\right) / \Delta \Theta$ with $\partial Z_{\min } / \partial t \geq 0$ in the present cases. At the right boundary, $\tilde{v}_{\xi}$ is positive (an outgoing wave from left to right), because at $\xi=1$ the velocity becomes $\tilde{v}_{\xi}=-\left(\partial Z_{\max } / \partial t\right) / \Delta \Theta$ with $\partial Z_{\max } / \partial t \leq 0$ in the present cases. For evolution of $Z_{\min }$ and $Z_{\max }$ in the present cases, see Fig. 5.
} 
brief description is given here. The DNSs are two-dimensional and carried out in a constant volume, achieved via periodic boundary conditions. As in a number of other DNS studies of ignition [20-34] the two-dimensional assumption was unfortunately necessary due to the computational expense involved of carrying out DNS in 3D with a 58 species mechanism, and considering a parametric study was carried out. Two-dimensional turbulence differs from 3D in that the vortex stretching mechanism is absent, and this should be borne in mind when interpreting the study. Despite this difference, previous comparisons of $2 \mathrm{D}$ and 3D DNS in the related problem of thermally stratified ignition nonetheless revealed only minor differences in terms of ignition behaviours [36]. Also as already mentioned, our DNS, like almost all others modelling ignition in engine-relevant thermochemical conditions, do not consider any large-scale flow geometry features, e.g. such as large-scale stratification arising from the fuel jet structure, tumble, swirl, etc. This is a necessary limitation at the present time due to the scale-separation inherent in practical conditions between large-scale features and the dissipative features that must be resolved in DNS.

The initial conditions for turbulence and mixture-fraction are random fields that are statistically homogeneous and isotropic having fluctuations generated from a prescribed spectrum. Local temperatures are specified as a function of mixture-fraction described later. A plot of initial mixture-fraction and vorticity magnitude in case HS3 (see Table 1) is provided in Fig. 2. The calculations evolve from their initial conditions with isotropic decaying turbulence distorting the fields of mixture-fraction, resulting in ignition occurring initially at isolated kernels, which then grow and merge before finally the last remaining pockets of reactants are burned.

S3D, an explicit DNS solver developed in Fortran language at the Combustion Research Facility at Sandia National Laboratories is used [88]. The S3D results are used as a reference to validate the $\mathrm{OF}-\mathrm{CMC}$ results. Also, the data are utilised for post-processing purposes to validate several modelling hypotheses relevant to the study. S3D is a high-order yet lowdissipation numerical solver. It utilises a Cartesian grid to solve for Navier-Stokes, species transport and total energy equations. The governing equations are discretised using a central, $8^{\text {th }}$ order finite differencing scheme for spatial derivatives and a 6 stage, $4^{\text {th }}$ order explicit Runge-Kutta scheme for time integration [89]. The convective term in transport equations are discretised using the skew-symmetric scheme [90] to reduce aliasing errors. CHEMKIN software libraries [91] are linked to S3D to evaluate thermo-physical properties and reaction rates. The same transport model as in $\mathrm{OF}-\mathrm{CMC}$ is implemented in $\mathrm{S} 3 \mathrm{D}$ for the present study.

Table 1 Parameters of simulated cases

\begin{tabular}{lllllll}
\hline Case & $\begin{array}{l}T_{0} \\
(\mathrm{~K})\end{array}$ & $\begin{array}{l}T^{\prime} \\
(\mathrm{K})\end{array}$ & $Z_{0}$ & $Z^{\prime}$ & $n_{\text {grid }}$ & $\phi_{0}$ \\
\hline LS1 & 850 & 15 & 0.0195 & 0.005 & 640 & 0.3 \\
LS2 & 950 & 15 & 0.0195 & 0.005 & 640 & 0.3 \\
LS3 & 1000 & 15 & 0.0195 & 0.005 & 640 & 0.3 \\
HS1 & 850 & 60 & 0.0195 & 0.02 & 1280 & 0.3 \\
HS2 & 950 & 60 & 0.0195 & 0.02 & 1280 & 0.3 \\
HS3 & 1000 & 60 & 0.0195 & 0.02 & 1280 & 0.3 \\
\hline
\end{tabular}


a

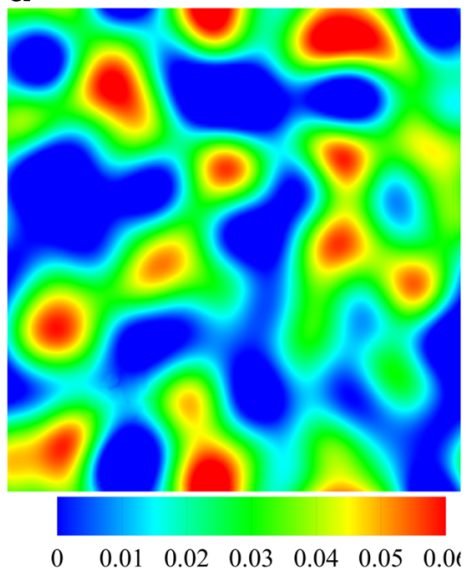

b

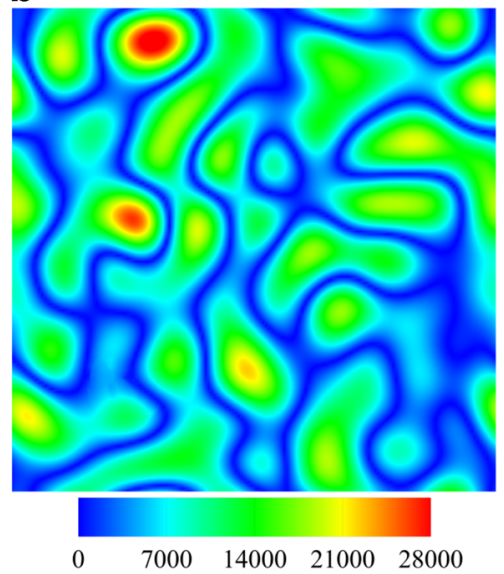

Fig. 2 Initial conditions for case HS3; (a) mixture-fraction; (b) vorticity magnitude expressed in 1/s

\subsection{Test cases and initial conditions}

Six two-dimensional test cases, with details outlined in Table 1, are simulated using S3D and OF-CMC. Initial conditions for these cases are taken from Ref. [29]. The only difference between DNSs here and those used in Ref. [29] is that here Le = 1 in the transport model, to be consistent with derivation of the CMC equation and the transport model used in the OFCMC implementation. The unity Lewis number is adopted in the DNS (as well as the CMC model) because the intention of the modelling is for practical application at a Reynolds number that is much higher than achievable with DNS. At high Reynolds number it is well known that the unity Lewis number formulation provides a more accurate picture of scalar transport behaviour than detailed models, e.g. [92]. Similar to Ref. [29], a reduced chemical mechanism for $n$-heptane/air with 58 species and 387 reactions developed for HCCI-like conditions is employed [93].

The cases in this study represent the conditions in the bulk gas around top dead centre away from the walls. Three mean temperatures of $850 \mathrm{~K}, 950 \mathrm{~K}$, and $1000 \mathrm{~K}$ are chosen to initialise the temperature field. These temperatures correspond to the negative temperature coefficient (NTC) regime of a homogeneous mixture of $n$-heptane/air with a mean fuel-air equivalence ratio of $\phi=0.3$ and a pressure of $p=40 \mathrm{~atm}$ [93]. Three cases are set up to have low stratification levels (LS1-3) in composition and temperature, and the other three have high stratification levels (HS1-3). All six cases have the same initial mean mixture-fraction ${ }^{6}$, with the mean mixture fraction set at $Z_{0}=$ 0.0195 corresponding to $\phi=0.3$. This choice of equivalence ratio is consistent with lean operating conditions, which is typical of HCCI/SCCI combustion. The low stratification cases have an RMS fluctuation of mixture-fraction that is approximately one quarter of the mean mixture fraction, while the high stratification cases have fluctuations that are the same order as the mean. These two sets of cases are selected to expose

\footnotetext{
${ }^{6}$ As per Ref. [28, 29], if negative mixture-fractions are encountered upon initialisation, they are truncated to zero. In the HS cases, this operation shifts the mean mixture-fraction from $Z_{0}=0.0195$ to $Z_{0}=0.0210$.
} 
different regimes of model performance in order to determine limits and increase of applicability.

All cases are initialised with the temperature specified according to a decreasing function of mixture fraction, which accounts for both fuel evaporation cooling and lower compression heating of higher fuel-concentration mixtures as a result of their lower ratio of specific heats. The function was determined in Ref. [29] by running a multi-zone model of an SCCI engine from the time of injection up to top dead centre, with an assumed Gaussian distribution of mixture fraction injected as liquid fuel at 300 crank angle degrees (where 360 is top dead centre). The fuel was first evaporated at constant volume and then the mixture was compressed up to TDC according to the slider-crank relationship. Because of the relatively small range of mixture fractions present here (unlike in a conventional diesel engine, for instance), the temperature dependence on mixture fraction was found to be approximately linear in the multi-zone model, so a linear mapping was adopted. It was also relatively independent of the amount of stratification and mean temperature, with the slope found to be $\left(T^{\prime} / Z^{\prime}=60 \mathrm{~K} / 0.02\right)$. This linear mapping is therefore applied to all cases.

To keep the computational cost low for DNS, considering the size of the chemical mechanism, a domain size of $3.2 \times 3.2 \mathrm{~mm}^{2}$ is chosen. The grid spacing is set at $640 \times 640$ for the LS cases and $1280 \times 1280$ for the HS cases. Periodic boundary conditions are imposed in all directions. The velocity field is initialised using a two-dimensional Passot-Pouquet spectrum for the turbulent kinetic energy [94]. The most energetic turbulent length scale, $l_{e}$, is set to $1 \mathrm{~mm}$ and the computational domain covers at least $3 l_{e}$. The turbulence time scale is set to $1 \mathrm{~ms}$ which corresponds to about $40 \%$ of the homogeneous ignition delay time obtained from the mean temperatures and equivalence ratios present in Table 1. This leads to $1 \mathrm{~m} / \mathrm{s}$ for the root mean square (RMS) of velocity field. All six cases have the same initial velocity field. This choice of length and time scales for turbulence initialisation allows a significant interaction between chemistry and turbulence, while it is still quite relevant to the TDC conditions in a realistic SCCI engine. For more information on justification of the initial conditions for turbulence, chemistry, and temperature with regard to SCCI conditions, the interested reader is referred to Ref. [29].

\section{Results and Discussion}

In this section the results are presented and discussed under two main subsections. First, the results of the hybrid DNS-CMC a posteriori method, which are obtained using the firstorder OF-CMC, are compared against the DNS data. In the second subsection, a priori analysis of the DNS data is performed to gain insight on the observed behaviour in the hybrid DNS-CMCa posteriori analysis and to provide directions for improved modelling of high stratification cases.

\subsection{The a posteriori analysis}

Here the results obtained from the OF-CMC solver are compared with the DNS data. At first, the mean heat release rates (HRRs) for all six cases are presented. Then deviations of the OF-CMC results from DNS are quantified based on the mean HRR. Afterwards, the same comparison is presented for temperature to reveal the deviations of the model from DNS. The results are then used to explain the possible reasons for disagreement in some of the cases considered here. 

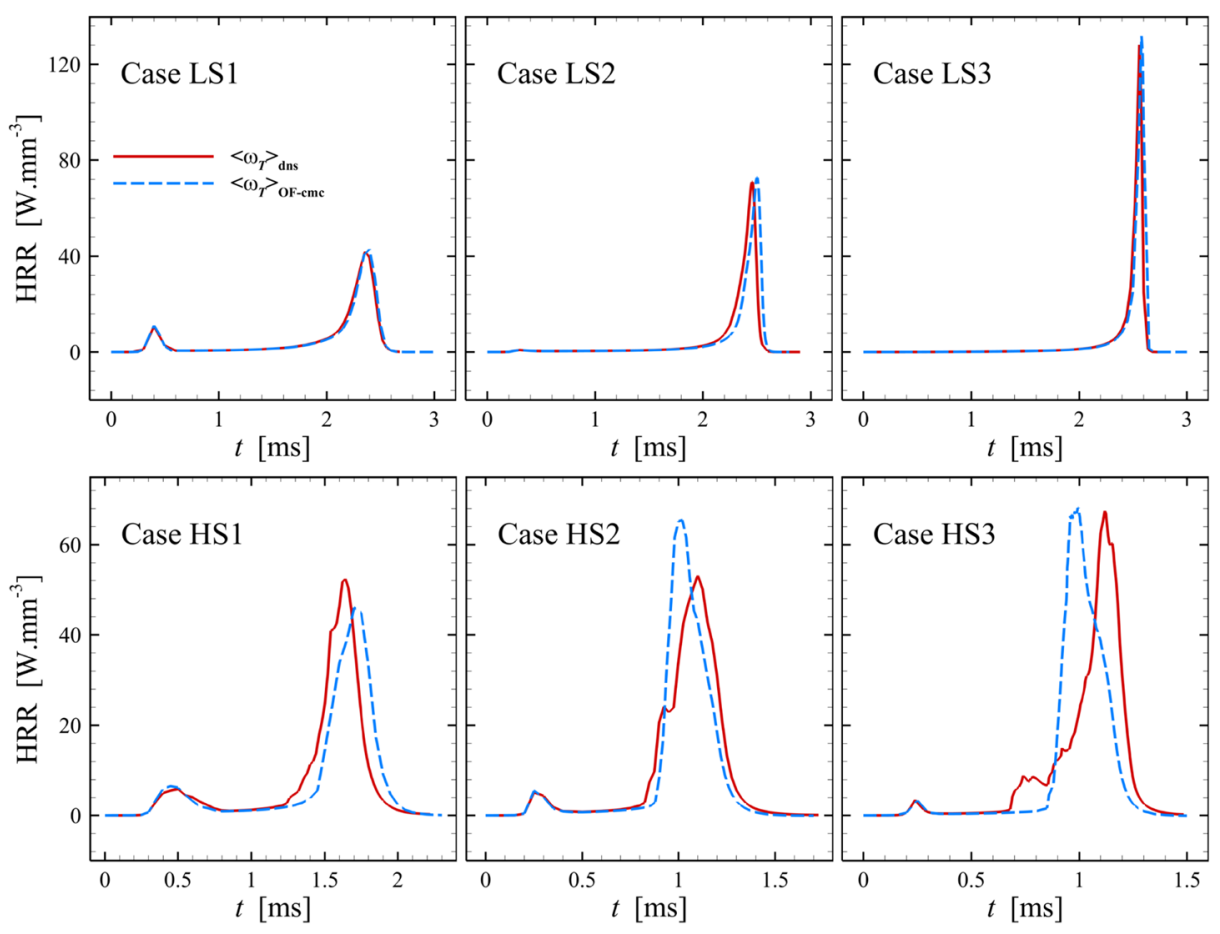

Fig. 3 Mean heat release rates as obtained from OF-CMC and DNS

\subsubsection{Autoignition using the OF-CMC}

To evaluate the performance of the OF-CMC implementation, the HRR is used as a reference for comparison to the DNS data. Results for all cases (Table 1) are presented in Fig. 3. In this figure, the HRR from the DNS, $\left\langle\dot{\omega}_{T}\right\rangle_{\mathrm{dns}}$, is compared with the HRR obtained from the OF-CMC, $\left\langle\dot{\omega}_{T}\right\rangle_{\mathrm{OF}-\mathrm{cmc}}$. As may be observed, in the LS cases, the first-order closure hypothesis works very well. In the HS cases, however, the OF-CMC results deviate from the DNS. These trends are similar to what has been observed in previous work on thermally stratified ignitions $[67,68]$.

To further quantify the deviation of the $\left\langle\dot{\omega}_{T}\right\rangle_{\mathrm{OF}-\mathrm{cmc}}$ from the $\left\langle\dot{\omega}_{T}\right\rangle_{\mathrm{dns}}$, the combustion timing and the combustion duration are used. The variable $\tau_{x} \%$ is defined as the time at which $x \%$ of the total heat is released. The ignition timing is quantified by $\tau_{10 \%}$, while the main combustion phasing is quantified by $\tau_{50 \%}$. The combustion duration, $\tau_{\mathrm{dur}}$, is defined as the difference between $\tau_{90 \%}$ and $\tau_{10 \%}$. In Fig. 4 , the relative errors ${ }^{7}$ in percentage for $\tau_{10 \%}, \tau_{50 \%}$ and $\tau_{\mathrm{dur}}$, reflecting the deviations of $\left\langle\dot{\omega}_{T}\right\rangle_{\mathrm{OF}-\mathrm{cmc}}$ from $\left\langle\dot{\omega}_{T}\right\rangle_{\mathrm{dns}}$, are illustrated.

Similar to the observation in Fig. 3, it can be seen that the LS cases demonstrate an excellent agreement with DNS both in terms of $\tau_{\text {ig }}$ and $\tau_{\text {dur }}$. The HS cases, however, demonstrate

\footnotetext{
${ }^{7}$ For example the relative error in $\tau_{10 \%}$ is found by the difference in $\tau_{10 \%}$ obtained from OF-CMC and DNS divided by $\tau_{10 \%}$ from DNS. The colours in Fig. 4 distinguish the positive values from negatives.
} 


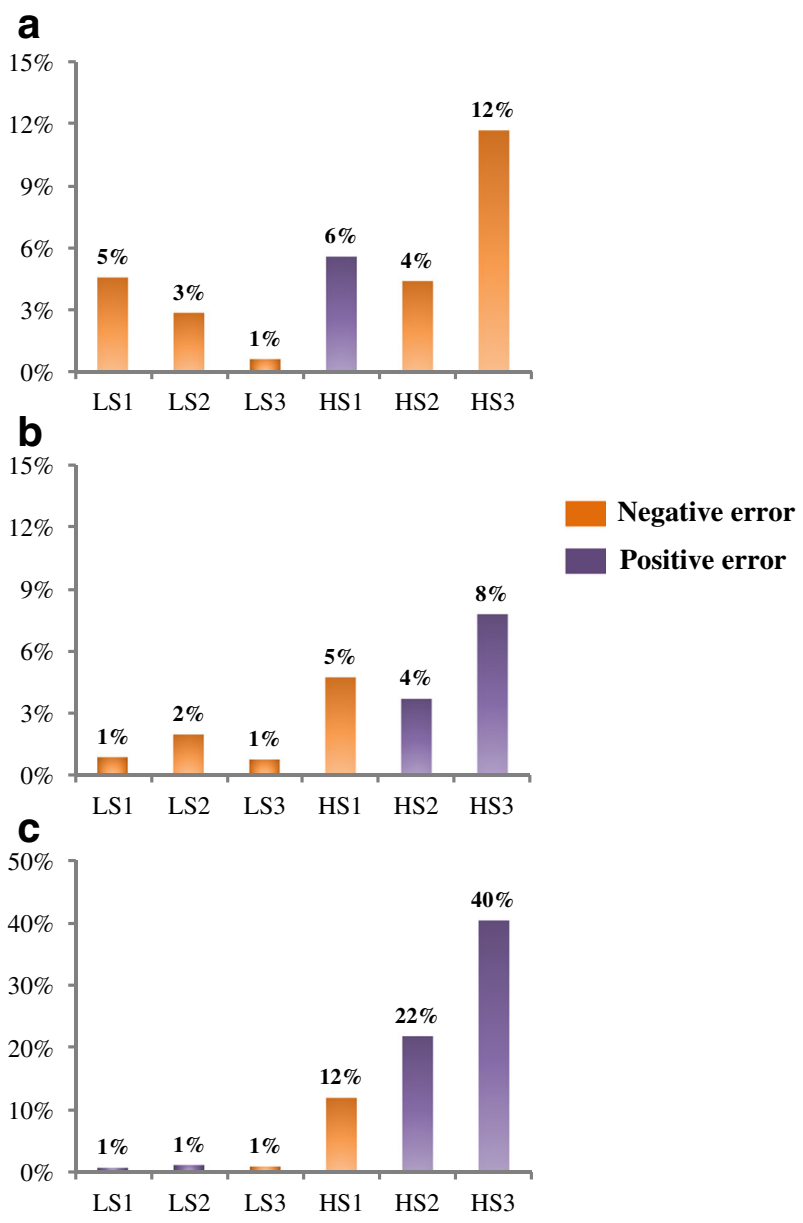

Fig. 4 Comparison of OF-CMC results to DNS; percentage of relative error in (a) ignition timing (10 percent of total heat release); (b) combustion phasing (50 percent of total heat release); (c) combustion duration. Negative error indicates that $\tau_{\mathrm{dns}}<\tau_{\mathrm{OF}-\mathrm{cmc}}$

higher levels of deviation, in general. The case HS1 has the best agreement among the HS cases, and the case HS3 exhibits the worst agreement. The disagreement of the case HS3 is large with $40 \%$ of the relative error in $\tau_{\text {dur }}$.

\subsubsection{Conditional temperature}

In Fig. 5, the conditional Favre-mean (CFM) temperature is presented as a function of mixture-fraction and time for the DNS and CMC models. The temperature's conditional root mean square (RMS), obtained from DNS, is also presented as third row. The Case LS1 shows the best agreement for the CFM of temperature. This is expected, since the temperature's conditional RMS for this case is negligible (see Fig. 5g). This shows that for case LS1, not only at the initial time are the reactive scalars strictly a function of mixture-fraction, 

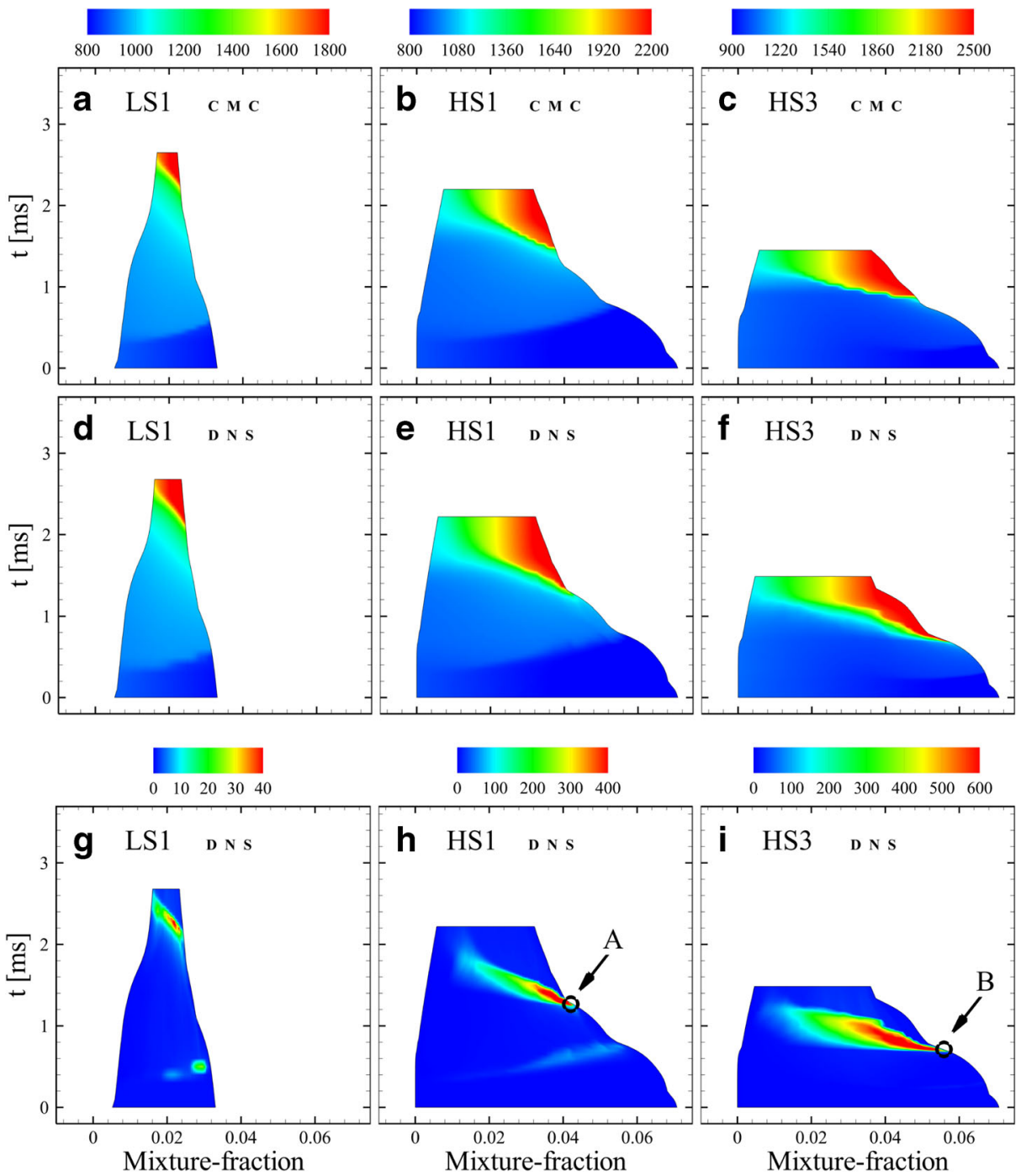

Fig. 5 Temperature evolution in mixture-fraction space; top row: conditional Favre mean of temperature from OF-CMC; middle row: conditional Favre mean of temperature from DNS; bottom row: conditional Favre fluctuations (rms) of temperature from DNS

but also throughout the combustion duration they remain highly dependent functions of mixture-fraction.

Case HS1 in Fig. 5.h exhibits a ten-fold higher RMS compared to that in case LS1. The fluctuations, in the second stage of autoignition, start from the richest mixture-fraction (point A) and very soon they disappear. For case HS3, however, as can be seen from Fig. 5.i, the fluctuations starting from point B persist for a long time and over a wider range of mixture-fractions. In both cases major fluctuations start on the richer side and penetrate into leaner mixtures at later times. This is because the second stage of heat release in these cases is initiated by autoignition in the relatively richer regions [29]. 


\subsubsection{Temperature field and statistics}

To further understand the reasons for the discrepancy between the OF-CMC and the DNS, Figs. 6 and 7 examine the temperature in physical and conditional space for the worst case for the model, case HS3. Figures 6 and 7 present results for a series of times spaced by $200 \mu$ s, with Fig. 6 covering the initial condition through the first stage ignition, and Fig. 7 covering the second stage ignition. In each figure, the top row shows, versus mixturefraction: the DNS temperatures; the DNS conditional mean temperature, and the OF-CMC temperature. The middle and lower rows show on a colour scale the temperature in physical space from the DNS and OF-CMC, respectively. Superimposed is a contour of $Z=0.035$, selected because this is a location where significant conditional fluctuations occur (c.f. Fig. 5.i).

Considering first Fig. 6, a quite good level of agreement for the conditional means is observed through the first stage of ignition; however, by $0.6 \mathrm{~ms}$ some conditional fluctuations are beginning to appear, as seen in the top row showing the DNS local temperatures. More significant discrepancies are observed by $0.8 \mathrm{~ms}$ in Fig. 7. Here the temperatures in the DNS show a clearly bimodal behaviour in mixture-fraction space (top row), with some regions having fully ignited while other regions remain at low temperatures. In physical space this can be observed as well, as shown in the middle and bottom rows. Here, the region marked $A_{1}$ in the DNS has fully ignited while the corresponding region $A_{2}$ in the OF-CMC

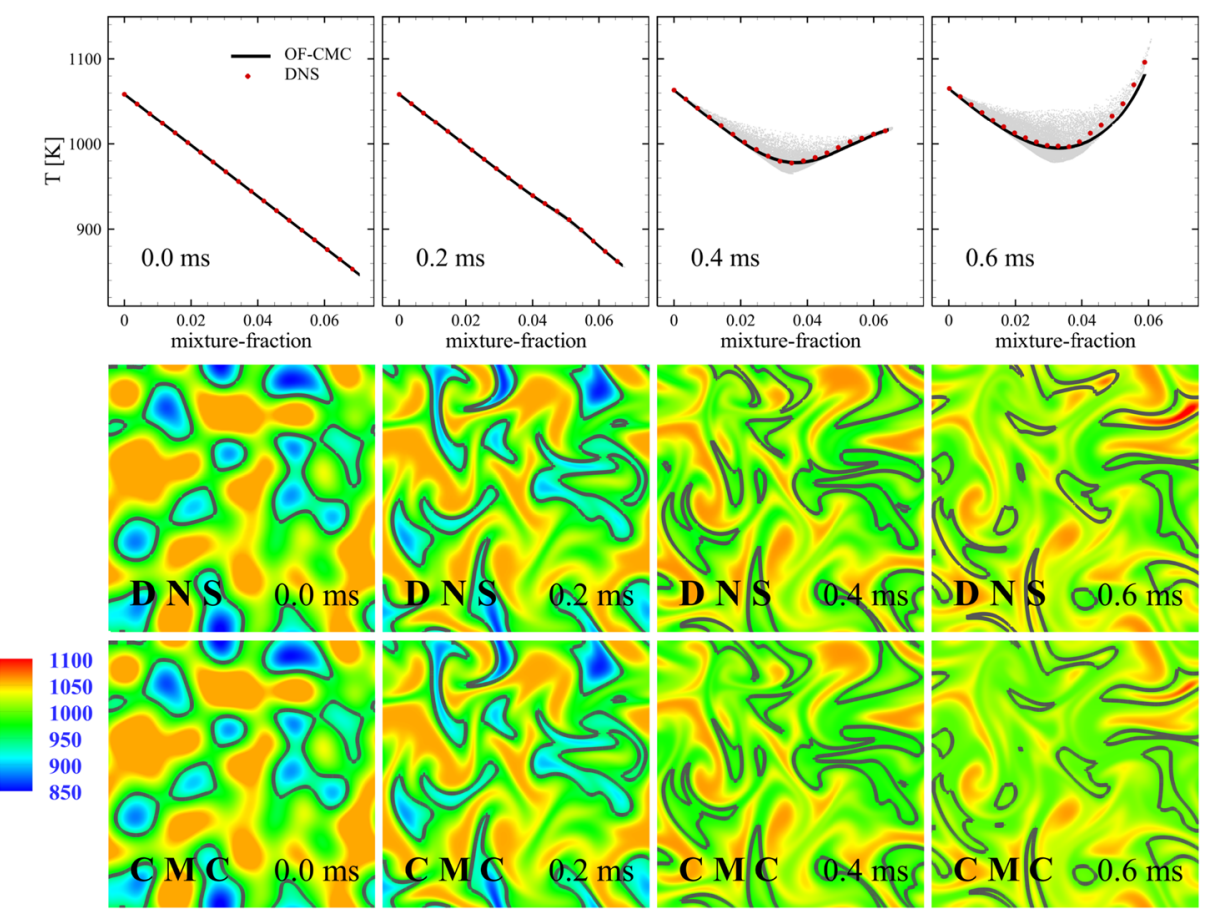

Fig. 6 Temperature for case HS3 during first stage of autoignition at times $t=[0,0.2,0.4,0.6] \mathrm{ms}$ from left to right; top row: conditional mean of temperature obtained from OF-CMC is compared to $T_{\eta}$ from DNS. The scatter plots of DNS temperature is shown on the background as reference; middle row: temperature contours and iso- $Z$ contour lines of $Z=0.035$ obtained from DNS; bottom row: the same contours for $\mathrm{OF}-\mathrm{CMC}$ results 


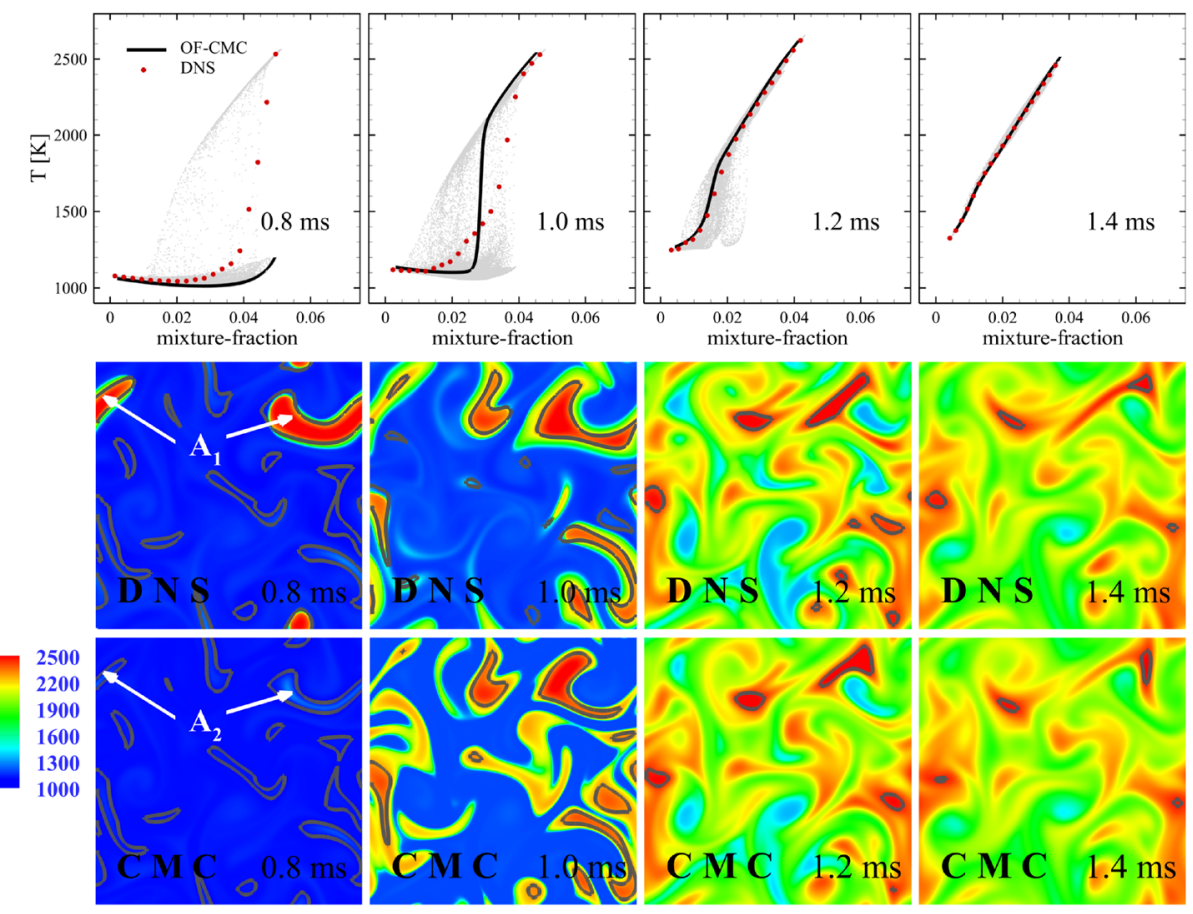

Fig. 7 Temperature for case HS3 during second stage of autoignition at times $t=[0.8,1.0,1.2,1.4] \mathrm{ms}$ from left to right; top row: conditional mean of temperature obtained from OF-CMC is compared to $T_{\eta}$ from DNS. The scatter plots of DNS temperature is shown on the background as reference; middle row: temperature contours and iso- $Z$ contour lines of $Z=0.035$ obtained from DNS; bottom row: the same contours for $\mathrm{OF}-\mathrm{CMC}$ results

is yet to ignite. At $1.0 \mathrm{~ms}$ the bimodal nature is even more apparent in the DNS; however at this time the CMC is advanced compared with the DNS on the relatively richer side, but lags on the relatively leaner side. At later times, the bimodality reduces in the DNS as a fully burned state is reached by all mixtures, and the agreement between DNS and CMC improves.

Previous work [29, 67, 68, 95, 96] has suggested that fluctuations of dissipation rate can alter local autoignition timing and hence generate conditional fluctuations. This hypothesis is investigated in Fig. 8 showing a sequence of the DNS temperatures, coloured by dissipation rate, versus mixture fraction at times from 0.4 to $0.8 \mathrm{~ms}$ (note the different temperature scale for $0.8 \mathrm{~ms}$ ). The figure clearly shows that higher dissipation rates correspond to more advanced reaction progress during this period. Although higher dissipation rate is generally found to retard ignition in the literature, recent work [96] has shown this is not always the case, since mixing from early igniting regions (here, on the rich side) can accelerate the ignition of later igniting regions (here, towards the learner side). The results confirm this phenomenon in the present conditions, and demonstrate that conditional fluctuations are generated by fluctuating dissipation rates.

It is important to note that the present results where scalar dissipation rate enhances reaction progress of intermediate mixtures are not contrary to prior literature on nonpremixed ignitions, where high dissipation rate delays progress of the first igniting kernels [95, 97, 


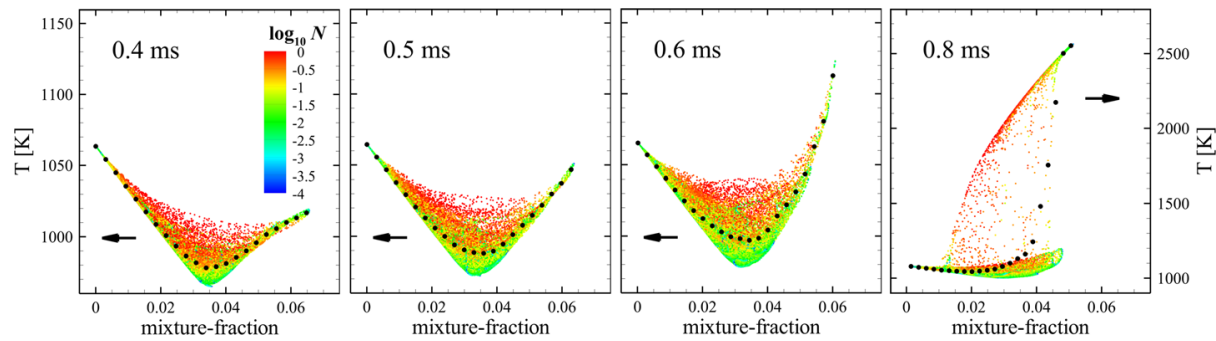

Fig. 8 Scatter plot of temperature versus mixture-fraction coloured by $\log _{10}$ of SDR for case HS3 in transition from first to the second stage of autoignition

98]. In most prior nonpremixed ignition studies, the most reactive mixture fraction, i.e. that with the shortest ignition delay, was found within the domain. In the present study, however, the most-reactive mixture fraction, if all mixtures were considered, is richer than the richest mixture within the domain. As a result, in the present cases shown in Figs. 6 and 8, ignition occurs first at the richest mixture in the domain. Because these richest points are isolated maxima of mixture fraction, their scalar dissipation always goes to zero. As a result, there are minimal scalar dissipation fluctuations in these regions and thus no significant effect of lower dissipation causing retarded ignition. After the kernels are established, however, higher dissipation rate on average tends to promote mixing into leaner regions, which accelerates the ignition in those regions, thus leading to the trends observed in Figs. 7 and 8.

In summary for this section, the results show that good performance can be obtained with the first order CMC for sufficiently low stratification, but at higher stratification the performance breaks down as a result of dissipation fluctuations generating conditional fluctuations in the composition state vector. The consequences for this physical phenomenon on the first-order conditioning closure and alternative advanced closures are investigated in the next section.

\subsection{The a priori analysis}

To provide insight into the reasons behind the deviations of the first-order OF-CMC from the DNS, in this section the CMC model and the underlying assumptions used in developing this model are verified using the DNS data. Remedies are then proposed within the a priori framework for future modelling directions. The structure of this section is as follows. The first-order closure hypothesis is evaluated using the DNS data. The second-order closure is investigated afterwards. Finally double conditioning is shown to provide an effective closure for the present cases.

\subsubsection{First-order closure}

As discussed in the previous section, the reaction rate term plays a crucial role in the evolution of species. Unlike the PDF-based methods where the reaction rate term appears in a closed form, in the $\mathrm{CMC}$ approach the conditional reaction rate term is in an unclosed form. The OF-CMC implementation of this equation is based on the first-order closure hypothesis, that is $\dot{\omega}_{\eta}=\dot{\omega}(\phi)$ where $\phi=\left[T_{\eta}, Y_{1, \eta}, Y_{2, \eta}, \cdots, Y_{N_{s}, \eta}, p_{\eta}\right]$. To assess the hypothesis for the present cases, the DNS data is used in a priori manner to calculate the reaction rates using first-order assumption. By utilising the PDF of the mixture-fraction, the mean 
reaction rates are calculated and consequently the volumetric mean of HRR, $\left\langle\dot{\omega}_{T}\right\rangle_{\mathrm{dns}, 1 \mathrm{st}}$, are obtained. The results for the HS cases are depicted in Fig. 9.

Interestingly, very poor agreement between $\left\langle\dot{\omega}_{T}\right\rangle_{\mathrm{dns}}, 1 \mathrm{st}$ and $\left\langle\dot{\omega}_{T}\right\rangle_{\mathrm{dns}}$ are observed in Fig. 9. It should be noted that for case HS3, the first-order assumption, leads to 30 times of overprediction in the mean HRR which is an extremely high level of disagreement. Referring back to the top row of Fig. 7, the reason for this breakdown is quite clear. The DNS shows a strongly bimodal behaviour where the most likely states on the upper and lower branches are not close to the conditional mean. Interestingly, the hybrid DNS-CMC a posteriori result from the OF-CMC is actually a lot better than expected from the a priori analysis; this is because the hybrid DNS-CMC a posteriori solution evolves along a physically realistic (if over-constrained) manifold, while the conditional average state does not necessarily lie on a physically realistic manifold [99], thus resulting in unphysically fast reaction rates.

\subsubsection{Second-order closure}

In an attempt to find a solution for the problem of closure of reaction rate term, second-order closure in an a priori manner is examined. A state vector, $\phi$, is defined for the conditional means of temperature, mass fractions, and pressure as

$$
\phi=\left[T_{\eta}, Y_{1, \eta}, Y_{2, \eta}, \cdots, Y_{N_{s}, \eta}, p_{\eta}\right]
$$

where $N_{s}$ is number of species. Also a state field, $\phi^{\prime \prime}$, is defined for corresponding conditional fluctuations as

$$
\phi^{\prime \prime}=\left[T^{\prime \prime}, Y_{1}^{\prime \prime}, Y_{2}^{\prime \prime}, \cdots, Y_{N_{s}}^{\prime \prime}, p^{\prime \prime}\right] .
$$

Taylor series expansion of reaction rate function about $\phi$ results in second-order closure of reaction rate, viz.

$$
\langle\dot{\omega} \mid \eta\rangle=\dot{\omega}(\phi)+\left.\frac{1}{2} \sum_{i=1}^{N_{s}+2} \sum_{j=1}^{N_{s}+2}\left\langle\phi_{i}^{\prime \prime} \phi_{j}^{\prime \prime} \mid \eta\right\rangle \frac{\partial^{2} \dot{\omega}}{\partial \phi_{i} \partial \phi_{j}}\right|_{\phi}+\mathcal{O}\left(\left\langle\phi_{i}^{\prime \prime} \phi_{j}^{\prime \prime} \phi_{k}^{\prime \prime} \mid \eta\right\rangle\right) .
$$

This second-order approach to estimate the reaction rates is implemented as an a priori analysis of the DNS. To ensure the implementation accuracy of Eq. 9, the improvements in reaction rate predictions using the second-order closure with respect to the first-order

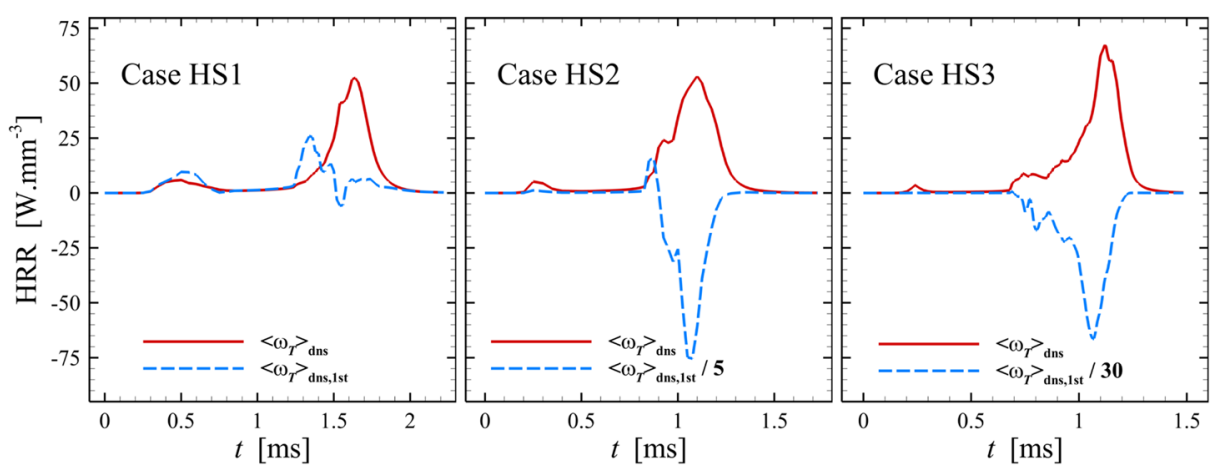

Fig. 9 heat release rates for the HS cases with first-order closure for reaction rates 

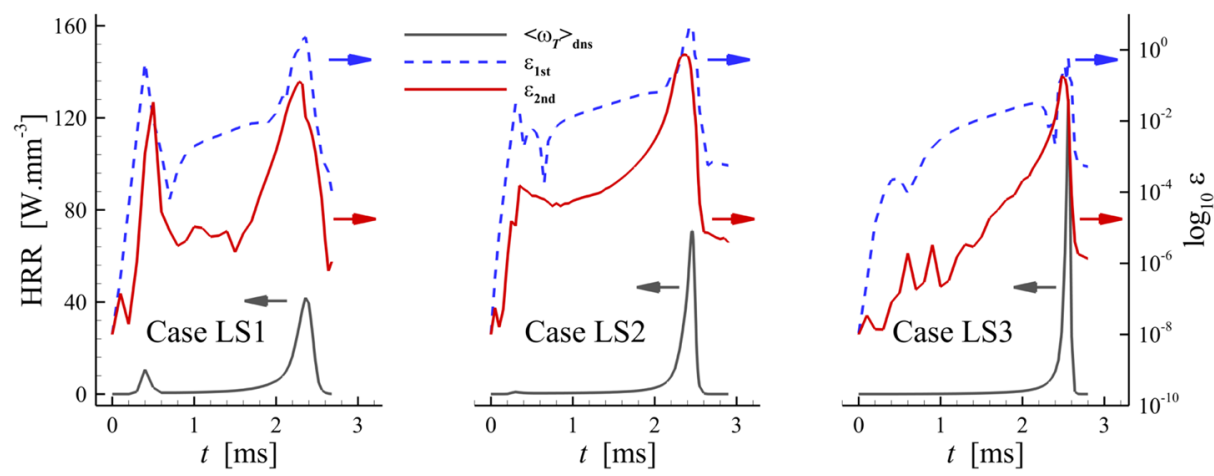

Fig. 10 Validation of the second-order closure implementation for the LS cases

closure are illustrated in Fig. 10. In this figure, $\varepsilon$ represents the absolute error in mean HRR, and it is defined as

and

$$
\varepsilon_{1 \mathrm{st}}=\left|\left\langle\dot{\omega}_{T}\right\rangle_{1 \mathrm{st}}-\left\langle\dot{\omega}_{T}\right\rangle_{\mathrm{dns}}\right|,
$$

$$
\varepsilon_{2 \text { nd }}=\left|\left\langle\dot{\omega}_{T}\right\rangle_{2 \text { nd }}-\left\langle\dot{\omega}_{T}\right\rangle_{\text {dns }}\right|,
$$

where $\left\langle\dot{\omega}_{T}\right\rangle_{2 \text { nd }}$ is the mean HRR obtained from PDF-weighted integral of the reaction rates estimated by Eq. 9. From Fig. 10 it is evident that, for all LS cases, $\varepsilon_{2 \text { nd }}$ is overall smaller than $\varepsilon_{1 \mathrm{st}}$. Of particular interest is the peaks of HRR which almost coincide with the peaks of $\varepsilon_{1 \text { st }}$. At the peak of $\varepsilon_{1 \mathrm{st}}$, the second-order closure reduces the error by almost a factor of 10 in all three LS cases. The results for the HS cases are presented ${ }^{8}$ in Fig. 11. By comparing these results to those of Fig. 9 for the first-order closure, it can be readily realised that the second-order closure not only does not improve the HRR estimations, but makes the agreement worse. This suggests that the second-order approach should not be the next step in the modelling direction for these particular cases.

The reason for failure of the second-order closure may be explained by reference to the conditional fluctuations demonstrated in scatter plots of Figs. 7 and 8 at $t=0.8 \mathrm{~ms}$. For simplicity of the discussion, let us assume the reaction rate is only a function of temperature, i.e. $\dot{\omega}=f(T)$. The second-order closure is achieved by a Taylor expansion ${ }^{9}$ around the conditional mean, for instance $T_{\eta} \simeq 1700 \mathrm{~K}$ in Fig. 7. The truncation error in this case, $\mathcal{O}\left(\left\langle\left[T-T_{\eta}\right]^{3} \mid \eta\right\rangle\right)$, would be small only if $T$ is close enough to $T_{\eta}$. However, Fig. 7 reveals that most of the statistics at around $t=0.8 \mathrm{~ms}$ have a temperature far away from $T_{\eta} \simeq$ $1700 \mathrm{~K}$. In other words, the bimodal shape of the PDF, where states are either burning or not burning, is the reason for breakdown of the second-order closure. It should be noted that at this instance, the joint PDF of mixture-fraction and temperature (not shown here for brevity) has null population around $T_{\eta} \simeq 1700 \mathrm{~K}$, because of the bi-modal behaviour of the mixture.

To further explain why this bi-modal behaviour causes a significant breakdown, reference is made here to the well-known fact that reaction systems in combustion tend to evolve along

\footnotetext{
${ }^{8}$ The oscillations in the second-order results arise from statistical noise due to the finite sample. A larger sample would reduce this noise but it is unlikely to change the key findings.

${ }^{9}$ If $\dot{\omega}$ is only a function of $T$, then Eq. 9 is still mathematically valid and the $\langle\cdot \mid\rangle$ operator reduces to a normal averaging.
} 

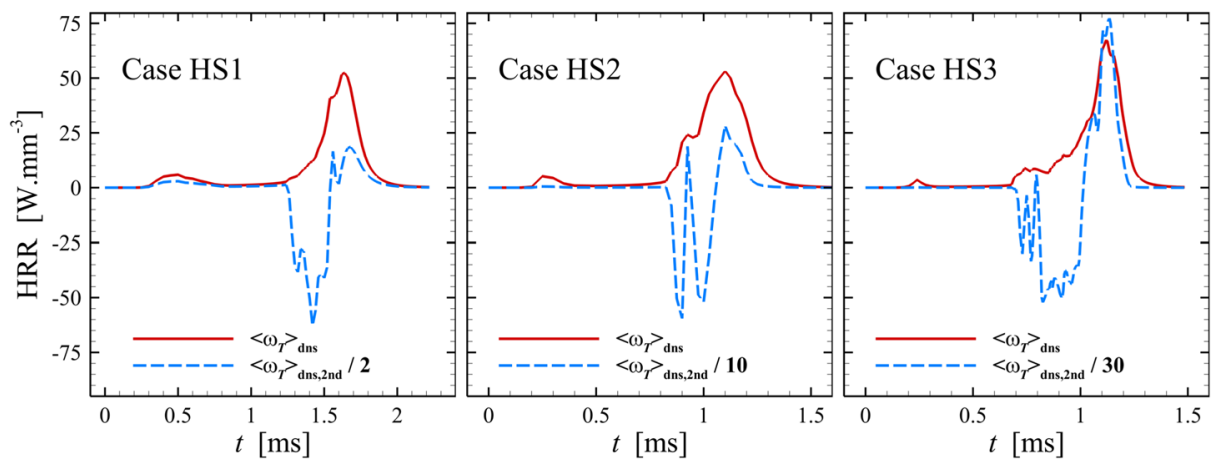

Fig. 11 heat release rates for the HS cases with second-order closure for reaction rates

low-dimensional manifolds, e.g. [99]. The rates of change of composition states along these manifolds are much lower than the rates of attraction to the manifold. Here, the averaging operation between (essentially) the two modes of the conditional PDFs, which are on the slow manifold, creates unphysical states that are very far from the slow manifold. As a result, very large reaction rates can result, which is what is observed here. This would not be a problem of course if the manifolds were constrained to flat hyper-planes, but they are not.

From a pure mathematical perspective, higher orders of closure could possibly improve this poor agreement [100]; however, from modelling point of view, this is not a practical direction to follow. In the present chemical mechanism, there are 58 species which means $\phi$ vector has 60 components (58 species + temperature + pressure) in each bin. Ignoring the diagonal elements, the Hessian matrix, $\partial^{2} \dot{\omega} /\left.\partial \phi_{i} \partial \phi_{j}\right|_{\phi}$, currently has almost 1800 nonrepeating elements which computationally are very expensive. Even if the number of species are to be reduced to a few principal components, the selection should happen from a pool of almost 1800 covariances, $\left\langle\phi_{i}^{\prime \prime} \phi_{j}^{\prime \prime} \mid \eta\right\rangle$. If a third order closure is attempted, this number would be even much higher, which makes modelling covariances and triple correlations a very challenging task, if not impossible. It should be noted that the failure here of second-order closure is believed to be a consequence of the highly bimodal conditional PDFs. In other circumstances, e.g. when there are significant conditional fluctuations but the conditional $\mathrm{PDF}$ is mono-modal about the conditional mean, higher order closures may prove more successful.

\subsubsection{First-order closure by double conditioning}

The second conditioning variable should be chosen as either a direct measure of reaction progress, such as sensible enthalpy $h_{s}$ [78], or a variable that affects reaction progress, such as scalar dissipation rate [79]. Here, the former option is investigated.

In Fig. 12 results for the mean HRR using sensible enthalpy as the second conditioning variable are demonstrated. Figure 12 reveals that double conditioning on mixture-fraction, $Z$, and sensible enthalpy, $h_{s}$, using first-order closure can resolve the issue of reaction rate closure for all cases. In this figure, 50 bins are used in the mixture-fraction space, similar to the first and second-order approaches in Figs. 9 and 11. In sensible enthalpy direction, however, 50 and 5 bins are examined. While $50 \times 50$ bin arrangement in $Z$ and $h_{s}$ directions, respectively, has completely resolved the closure problem, the $50 \times 5$ bin arrangement is 

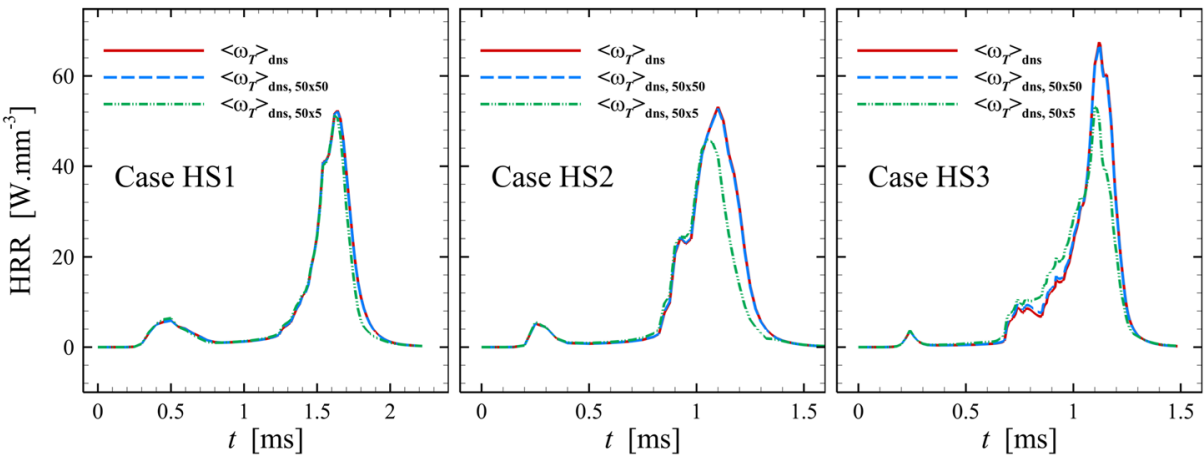

Fig. 12 heat release rates with first-order closure for reaction rates doubly conditioned on sensible enthalpy and mixture-fraction

also producing highly encouraging results ${ }^{10}$. This is expected, since combustion in the HS cases is highly bi-modal, where two extremes of hot and cold zones are formed during autoignition and as long as the two extremes are addressed, first-order closure should work.

\section{Concluding Remarks}

Spatially zero-dimensional conditional moment closure models for reaction rates were investigated to address the autoignition in $n$-heptane/air mixtures with composition stratifications under SCCI-like conditions. First-order closure, second-order closure, and double conditioning were contrasted as modelling options. A series of two-dimensional DNS were performed to generate the data necessary for a priori and hybrid DNS-CMC a posteriori analysis of the models. Two levels of stratifications with three different mean temperatures within the NTC regime of $n$-heptane were investigated. The first-order CMC model was implemented in OpenFOAM ${ }^{\circledR}$ and its performance was assessed by hybrid DNSCMC a posteriori comparison to the DNS data. To avoid any uncertainties associated with modelling the scalar dissipation rate, and to focus exclusively on the conditional moment closure assumptions, the CFD solution used the same grid resolution as DNS. Subsequently, the performance of first-order closure, second-order closure and double conditioning was compared using a priori tests. The following concluding remarks may be made.

- Excellent agreement between the first-order OF-CMC and DNS is observed for the low stratification cases. In the high stratification cases, while a strong disagreement between exact reaction rates from DNS and those obtained using the first-order closure in an $a$ priori study is observed, the hybrid DNS-CMC a posteriori OF-CMC predictions of reaction rates are not as bad. The relatively poorer performance in the a priori test is attributed to unphysical combinations of the state vector, which deviate significantly from physically occurring states that have a bimodal behaviour in conditional space.

- While second-order closure can improve reaction rate estimations for the LS cases, it can not offer improvement in the HS cases. This is attributed to high levels of conditional fluctuations with respect to mixture-fraction in the HS cases.

\footnotetext{
${ }^{10}$ In practice 5 bins are not enough and more is needed to capture the diffusive transport in the progress variable space which has to be accurately modelled.
} 
- Double conditioning with mixture-fraction and sensible enthalpy, however, provides a good first-order closure to the reaction rates. The analysis therefore suggests that double conditioning is a promising possibility for modelling SCCI combustion in the framework of CMC. However, the extra terms appearing in the doubly-CMC equation such as the dissipation rate of sensible enthalpy and cross-scalar dissipation term would need further modelling consideration, if this path is to be pursued [38].

Acknowledgements This work was supported by the Australian Research Council. The research benefited from computational resources provided through the National Computational Merit Allocation Scheme, supported by the Australian Government. The computational facilities supporting this project included the Australian NCI National Facility, the partner share of the NCI facility provided by Intersect Australia Pty Ltd., Pawsey Supercomputing Centre (with funding from the Australian Government and the Government of Western Australia), and the UNSW Faculty of Engineering.

\section{Compliance with Ethical Standards}

Funding This research was supported under Australian Research Council under the Discovery Projects funding scheme (project numbers DP110104763 and DP150104393) and the Linkage Infrastructure, Equipment and Facilities scheme (project numbers LE140100002, LE160100002 and LE160100051). Evatt Hawkes is the recipient of an Australian Research Council Future Fellowship (project number FT100100536).

Conflict of interests The authors declare that they have no conflict of interest.

\section{References}

1. Dec, J.E.: Proc. Combust. Inst. 32(2), 2727 (2009). http://www.sciencedirect.com/science/article/pii/ S1540748908001739

2. Yao, M., Zheng, Z., Liu, H.: Prog. Energy Combust. Sci. 35(5), 398 (2009). http://www.sciencedirect. com/science/article/pii/S0360128509000197

3. Lu, X., Han, D., Huang, Z.: Prog. Energy Combust. Sci. 37(6), 741 (2011). http://www.sciencedirect. com/science/article/pii/S0360128511000219

4. Saxena, S., Bedoya, I.D.: Prog. Energy Combust. Sci. 39(5), 457 (2013). http://www.sciencedirect.com/ science/article/pii/S0360128513000257

5. Yang, Y., Dec, J., Dronniou, N., Sjöberg, M., Cannella, W.: SAE Paper 2011-01-1359. http://papers.sae. org/2011-01-1359/ (2011)

6. Yang, Y., Dec, J.E., Dronniou, N., Sjöberg, M.: Proc. Combust. Inst. 33(2), 3047 (2011). http://www. sciencedirect.com/science/article/pii/S1540748910001999

7. Sjöberg, M., Dec, J.E.: SAE Paper 2006-04-03. http://papers.sae.org/2006-01-0629/ (2006)

8. Yang, Y., Dec, J., Dronniou, N., Cannella, W.: SAE Paper 2012-01-1120. http://papers.sae.org/ 2012-01-1120/ (2012)

9. Dec, J.E., Yang, Y., Dronniou, N.: SAE Paper 2011-01-0897. http://papers.sae.org/2011-01-0897/ (2011)

10. Dec, J.E., Sjöberg, M.: SAE Paper 2004-01-0557. http://papers.sae.org/2004-01-0557/ (2004)

11. Hwang, W., Dec, J.E., Sjöberg, M.: SAE Paper 2007-01-4130. http://papers.sae.org/2007-01-4130/ (2007)

12. Ma, J., Lü, X., Ji, L., Huang, Z.: Energy Fuels 22(2), 954 (2008). http://pubs.acs.org/doi/abs/10.1021/ ef700685v?journalCode=enfuem

13. Dahl, D., Andersson, M., Berntsson, A., Denbratt, I., Koopmans, L.: SAE Paper 2009-01-1785. http:// papers.sae.org/2009-01-1785/ (2009)

14. Wada, Y., Senda, J.: SAE Paper 2009-01-0498. http://papers.sae.org/2009-01-0498/ (2009)

15. Herold, R., Krasselt, J., Foster, D.E., Ghandhi, J., Reuss, D., Najt, P.: SAE Paper 2009-01-1106 2(1), 1034 (2009). http://papers.sae.org/2009-01-1106/

16. Krasselt, J., Foster, D.E., Ghandhi, J., Herold, R., Reuss, D., Najt, P.: SAE Paper 2009-01-1105. http:// papers.sae.org/2009-01-1105/ (2009) 
17. Kalghatgi, G., Hildingsson, L., Harrison, A., Johansson, B.: Proc. Combust. Inst. 33(2), 3015 (2011). http://www.sciencedirect.com/science/article/pii/S1540748910002361

18. Viggiano, A., Magi, V.: SAE Paper 2011-01-0837, pp .01-0837. http://papers.sae.org/2011-01-0837/ (2011)

19. Jung, D., Kwon, O., Lim, O.T., Mech, J.: Sci. Technol. 25(6), 1383 (2011). http://link.springer.com/ article/10.1007/s12206-011-0404-1

20. Chen, J.H., Hawkes, E.R., Sankaran, R., Mason, S.D., Im, H.G.: Combust. Flame 145(1), 128 (2006). http://www.sciencedirect.com/science/article/pii/S0010218005003342

21. Hawkes, E.R., Sankaran, R., Pébay, P.P., Chen, J.H.: Combust. Flame 145(1), 145 (2006). http://www. sciencedirect.com/science/article/pii/S0010218005003354

22. Yoo, C.S., Lu, T., Chen, J.H., Law, C.K.: Combust. Flame 158(9), 1727 (2011). http://www. sciencedirect.com/science/article/pii/S001021801100040X

23. Bhagatwala, A., Chen, J.H., Lu, T.: Combust. Flame 161(7), 1826 (2014). http://www.sciencedirect. com/science/article/pii/S0010218014000030

24. Fukushima, N., Katayama, M., Naka, Y., Oobayashi, T., Shimura, M., Nada, Y., Tanahashi, M., Miyauchi, T.: Proc. Combust. Inst. 35(3), 3009 (2015). http://www.sciencedirect.com/science/article/ pii/S1540748914003691

25. Yoo, C.S., Luo, Z., Lu, T., Kim, H., Chen, J.H.: Proc. Combust. Inst. 34(2), 2985 (2013). http://www. sciencedirect.com/science/article/pii/S154074891200020X

26. Luong, M.B., Luo, Z., Lu, T., Chung, S.H., Yoo, C.S.: Combust. Flame 160(10), 2038 (2013). http:// www.sciencedirect.com/science/article/pii/S0010218013001569

27. Kim, S.O., Luong, M.B., Chen, J.H., Yoo, C.S.: Combust Flame. http://www.sciencedirect.com/science/ article/pii/S0010218014002685 (2014)

28. Bansal, G., Im, H.G.: Combust. Flame 158(11), 2105 (2011). http://www.sciencedirect.com/science/ article/pii/S0010218011001064

29. Talei, M., Hawkes, E.R.: Proc. Combust. Inst. 35(3), 3027 (2015). http://www.sciencedirect.com/ science/article/pii/S1540748914004234

30. Luong, M.B., Yu, G.H., Lu, T., Chung, S.H., Yoo, C.S.: Combust. Flame 162(12), 4566 (2015). http:// www.sciencedirect.com/science/article/pii/S0010218015003181

31. Sun, W., Won, S.H., Gou, X., Ju, Y.: Proc. Combust. Inst. 35(1), 1049 (2015). http://www.sciencedirect. com/science/article/pii/S1540748914001448

32. El-Asrag, H.A., Ju, Y.: Combust. Theor. Model 17(2), 316 (2013). http://www.tandfonline.com/doi/abs/ 10.1080/13647830.2013.764020

33. El-Asrag, H.A., Ju, Y.: Combust. Flame 161(1), 256 (2014). http://www.sciencedirect.com/science/ article/pii/S0010218013002708

34. Luong, M.B., Lu, T., Chung, S.H., Yoo, C.S.: Combust. Flame 161(11), 2878 (2014). http://www. sciencedirect.com/science/article/pii/S0010218014001345

35. Bansal, G., Mascarenhas, A., Chen, J.H.: Combust. Flame 162(3), 688 (2015). http://www.sciencedirect. com/science/article/pii/S0010218014002697

36. Yu, R., Bai, X.S.: Combust. Flame 160(9), 1706 (2013). http://www.sciencedirect.com/science/article/ pii/S0010218013001259

37. Krisman, A., Hawkes, E.R., Kook, S., Sjöberg, M., Dec, J.E.: Fuel 99, 45 (2012). http://www. sciencedirect.com/science/article/pii/S0016236112002657

38. Mittal, V., Cook, D.J., Pitsch, H.: Combust. Flame 159(8), 2767 (2012). http://www.sciencedirect.com/ science/article/pii/S0010218012000284

39. Cook, D.J., Pitsch, H., Chen, J.H., Hawkes, E.R.: Proc. Combust. Inst. 31(2), 2903 (2007). http://www. sciencedirect.com/science/article/pii/S1540748906002422

40. Pal, P., Keum, S., Im, H.G.: Int. J. Engine Res, 280-290 (2015). http://jer.sagepub.com/content/17/3/280

41. Zhang, Y., Kung, E., Haworth, D.: Proc. Combust. Inst. 30(2), 2763 (2005). http://www.sciencedirect. com/science/article/pii/S0082078404002759

42. Bisetti, F., Chen, J.Y., Hawkes, E.R., Chen, J.H.: Combust. Flame 155(4), 571 (2008). http://www. sciencedirect.com/science/article/pii/S0010218008001818

43. Klimenko, A.Y.: Fluid Dyn. 25(3), 327 (1990). http://link.springer.com/article/10.1007/BF01049811

44. Bilger, R.: Phys. Fluids 5(2), 436 (1993). http://scitation.aip.org/content/aip/journal/pofa/5/2/10.1063/ 1.858867

45. Klimenko, A.Y., Bilger, R.W.: Prog. Energy Combust. Sci 25(6), 595 (1999). http://www.sciencedirect. com/science/article/pii/S0360128599000064

46. Navarro-Martinez, S., Kronenburg, A.: Proc. Combust. Inst. 31(2), 1721 (2007). http://www. sciencedirect.com/science/article/pii/S1540748906002136

47. Richardson, E., Mastorakos, E.: Proc. Medit. Comb. Symp. 5, (2007). http://eprints.soton.ac.uk/203183/ 
48. Kim, G., Kang, S., Kim, Y., Bilger, R., Cleary, M.: Combust. Theor. Model 11(4), 527 (2007). http:// www.tandfonline.com/doi/abs/10.1080/13647830600985297

49. Sreedhara, S., Huh, K.Y.: Combust. Flame 143(1), 119 (2005). http://www.sciencedirect.com/science/ article/pii/S0010218005001446

50. Kim, S.H., Huh, K.Y., Fraser, R.A.: Proc. Combust. Inst. 28(1), 185 (2000). http://www.sciencedirect. com/science/article/pii/S0082078400802106

51. Navarro-Martinez, S., Kronenburg, A.: Proc. Combust. Inst. 32(1), 1509 (2009). http://www. sciencedirect.com/science/article/pii/S1540748908001703

52. Devaud, C., Bray, K.: Combust. Flame 132(1), 102 (2003). http://www.sciencedirect.com/science/ article/pii/S0010218002004273

53. Sayed, A.E., Devaud, C.: Combust. Theor. Model 12(5), 943 (2008). http://www.tandfonline.com/doi/ abs/10.1080/13647830802116469

54. Buckrell, A., Devaud, C.: Flow Turb. Comb. 90(3), 621 (2013). http://link.springer.com/article/10.1007/ s10494-013-9445-0

55. Garmory, A., Mastorakos, E.: Proc. Combust. Inst. 33(1), 1673 (2011). http://www.sciencedirect.com/ science/article/pii/S1540748910001094

56. Triantafyllidis, A., Mastorakos, E.: Flow Turb. Comb. 84(3), 481 (2010). http://link.springer.com/article/ 10.1007/s10494-009-9226-y

57. De Paola, G., Kim, I., Mastorakos, E.: Flow Turb. Comb. 82(4), 455 (2009). http://link.springer.com/ article/10.1007/s10494-008-9183-X

58. Richardson, E., Yoo, C.S., Chen, J.: Proc. Combust. Inst. 32(2), 1695 (2009). http://www.sciencedirect. com/science/article/pii/S1540748908002216

59. Kim, S.H., Huh, K.Y.: Combust. Flame 138(4), 336 (2004). http://www.sciencedirect.com/science/ article/pii/S0010218004001336

60. Cleary, M., Kent, J., Bilger, R.: Proc. Combust. Inst. 29(1), 273 (2002). http://www.sciencedirect.com/ science/article/pii/S1540748902800372

61. Cleary, M., Kent, J.: Combust. Flame 143(4), 357 (2005). http://www.sciencedirect.com/science/article/ pii/S0010218005002233

62. Mantel, T., Bilger, R.: Combust. Sci. Technol. 110(1), 393 (1995). http://www.tandfonline.com/doi/abs/ $10.1080 / 00102209508951933$

63. Swaminathan, N., Bilger, R.: Combust. Theor. Model 5(2), 241 (2001). http://www.tandfonline.com/ doi/abs/10.1088/1364-7830/5/2/307

64. Amzin, S., Swaminathan, N., Rogerson, J., Kent, J.: Combust. Sci. Technol. 184(10-11), 1743 (2012). http://www.tandfonline.com/doi/abs/10.1080/00102202.2012.690629

65. Amzin, S., Swaminathan, N.: Combust. Theor. Model 17(6), 1125 (2013). http://www.tandfonline.com/ doi/abs/10.1080/13647830.2013.848382

66. Thornber, B., Bilger, R., Masri, A., Hawkes, E.: J. Comput. Phys. 230(20), 7687 (2011). http://www. sciencedirect.com/science/article/pii/S0021999111003974

67. Salehi, F., Talei, M., Hawkes, E.R., Yoo, C.S., Lucchini, T., D’Errico, G., Kook, S.: Proc. Combust. Inst. 35(3), 3087 (2015). http://www.sciencedirect.com/science/article/pii/S1540748914000388

68. Salehi, F., Talei, M., Hawkes, E.R., Yoo, C.S., Lucchini, T., D’Errico, G., Kook, S.: Flow Turb. Comb., 1-28 (2015). http://link.springer.com/article/10.1007/s10494-015-9604-6

69. Wright, Y., De Paola, G., Boulouchos, K., Mastorakos, E.: Combust. Flame 143(4), 402 (2005). http:// www.sciencedirect.com/science/article/pii/S0010218005002269

70. Bolla, M., Wright, Y.M., Boulouchos, K., Borghesi, G., Mastorakos, E.: Combust. Sci. Technol. 185(5), 766 (2013). http://www.tandfonline.com/doi/abs/10.1080/00102202.2012.752362

71. Han, I., Huh, K.Y.: Int. J. Automotive Tech 6, 571 (2005). http://www.erc.wisc.edu/documents/ 6-KIVAUsers_CMC_engine.pdf

72. De Paola, G., Mastorakos, E., Wright, Y., Boulouchos, K.: Combust. Sci. Technol. 180(5), 883 (2008). http://www.tandfonline.com/doi/abs/10.1080/00102200801894273\#.V0AnTJF96Uk

73. Wright, Y., Boulouchos, K., De Paola, G.: E. Mastorakos, SAE Paper 2009-01-0717 2(1), 714 (2009). http://papers.sae.org/2009-01-0717/

74. Seo, J., Lee, D., Huh, K.Y., Chung, J.: Combust. Sci. Technol. 182(9), 1241 (2010). http://www. tandfonline.com/doi/abs/10.1080/00102201003639300

75. Borghesi, G., Mastorakos, E., Devaud, C.B., Bilger, R.W.: Combust. Theor. Model 15(5), 725 (2011). http://www.tandfonline.com/doi/abs/10.1080/13647830.2011.560282\#.V0AmiJF96Uk

76. Bolla, M., Farrace, D., Wright, Y.M., Boulouchos, K.: Fuel 117, 309 (2014). http://www.sciencedirect. com/science/article/pii/S0016236113008843

77. Mastorakos, E., Bilger, R.: Phys. Fluids 10(6), 1246 (1998). http://scitation.aip.org/content/aip/journal/ pof $2 / 10 / 6 / 10.1063 / 1.869652$ 
78. Kronenburg, A.: Phys. Fluids 16(7), 2640 (2004). http://scitation.aip.org/content/aip/journal/pof2/16/7/ $10.1063 / 1.1758219$

79. Cha, C.M., Kosály, G., Pitsch, H.: Phys. Fluids 13(12), 3824 (2001). http://scitation.aip.org/content/aip/ journal/pof2/13/12/10.1063/1.1415426

80. Sreedhara, S., Lakshmisha, K.: Proc. Combust. Inst. 29(2), 2051 (2002). http://www.sciencedirect.com/ science/article/pii/S1540748902802504

81. Sreedhara, S., Lakshmisha, K.: Proc. Combust. Inst. 29(2), 2069 (2002). http://www.sciencedirect.com/ science/article/pii/S1540748902802528

82. Cao, S., Echekki, T.: Combust. Flame 151(1), 120 (2007). http://www.sciencedirect.com/science/article/ pii/S0010218007001101

83. Borghesi, G., Mastorakos, E., Cant, R.S.: Combust. Flame 160(7), 1254 (2013). http://www. sciencedirect.com/science/article/pii/S0010218013000515

84. Issa, R.I.: J. Comput. Phys. 62(1), 40 (1986). http://www.sciencedirect.com/science/article/pii/ 0021999186900999

85. Jasak, H.: Error analysis and estimation for the finite volume method with applications to fluid flows. Ph.D. thesis, Imperial College. http://ethos.bl.uk/OrderDetails.do?uin=uk.bl.ethos.311802 (1996)

86. Weller, H.G., Tabor, G., Jasak, H., Fureby, C.: Comp. Phys. 12(6), 620 (1998). http://scitation.aip.org/ content/aip/journal/cip/12/6/10.1063/1.168744

87. LeVeque, R.J.: Finite volume methods for hyperbolic problems, vol. 31. University Press, Cambridge (2002). https://books.google.com.au/books?id=mfAfAwAAQBAJ\&dq=Finite+Volume+Methods+for+ Hyperbolic+Problems\&lr=

88. Chen, J.H., Choudhary, A., De Supinski, B., DeVries, M., Hawkes, E., Klasky, S., Liao, W., Ma, K., Mellor-Crummey, J., Podhorszki, N., et al.: Comp. Sci. Discov. 2(1), 015001 (2009). http://iopscience. iop.org/article/10.1088/1749-4699/2/1/015001/meta

89. Kennedy, C.A., Carpenter, M.H., Lewis, R.M.: App. Num. Math. 35(3), 177 (2000). http://www. sciencedirect.com/science/article/pii/S0168927499001415

90. Kennedy, C.A., Gruber, A.: J. Comput. Phys. 227(3), 1676 (2008). http://www.sciencedirect.com/ science/article/pii/S0021999107004251

91. Kee, R., Dixon-Lewis, G., Warnatz, J., Coltrin, M., Miller, J.: Technical Report SAND86-8246, Sandia National Laboratories (1986)

92. Barlow, R., Frank, J., Karpetis, A., Chen, J.Y.: Combust. Flame 143(4), 433 (2005). http://www. sciencedirect.com/science/article/pii/S0010218005002282

93. Yoo, C.S., Lu, T., Chen, J.H., Law, C.K.: Combust. Flame 158(9), 1727 (2011). http://www. sciencedirect.com/science/article/pii/S001021801100040X

94. Hinze, J.: Turbulence. McGraw-Hill, New York (1975)

95. Mastorakos, E., Baritaud, T., Poinsot, T.: Combust. Flame 109(1), 198 (1997). http://www.sciencedirect. com/science/article/pii/S0010218096001496

96. Krisman, A., Hawkes, E.R., Talei, M., Bhagatwala, A., Chen, J.H.: A direct numerical simulation of cool-flame affected autoignition in diesel engine-relevant conditions. Submitted to the Proceedings of the Combustion Institute, Manuscript Number PROCI-D-15-01109R1 (2015)

97. Echekki, T., Chen, J.H.: Proc. Combust. Inst. 29(2), 2061 (2002). http://www.sciencedirect.com/science/ article/pii/S1540748902802516

98. Echekki, T., Chen, J.H.: Combust. Flame 134(3), 169 (2003). http://www.sciencedirect.com/science/ article/pii/S0010218003000889

99. Pope, S.B.: Proc. Combust. Inst. 34(1), 1 (2013). http://www.sciencedirect.com/science/article/pii/ S1540748912003963

100. Cha, C.M., Pitsch, H.: Combust. Theor. Model 6(3), 425 (2002). http://www.tandfonline.com/doi/abs/ $10.1088 / 1364-7830 / 6 / 3 / 303$ 